Review

\title{
Courier service for phosphatidylinositol: PITPs deliver on demand
}

\author{
Tim G. Ashlin, Nicholas J. Blunsom, Shamshad Cockcroft \\ Dept. of Neuroscience, Physiology and Pharmacology, Division of Biosciences, University College London, London WC1E 6JJ, UK
}

\section{A R T I C L E I N F O}

\section{Keywords:}

Phospholipase C

Phosphatidic acid

Lipid transfer

Phosphatidylinositol

PITPNC1

Golgi

Membrane traffic

\begin{abstract}
A B S T R A C T
Phosphatidylinositol is the parent lipid for the synthesis of seven phosphorylated inositol lipids and each of them play specific roles in numerous processes including receptor-mediated signalling, actin cytoskeleton dynamics and membrane trafficking. PI synthesis is localised to the endoplasmic reticulum (ER) whilst its phosphorylated derivatives are found in other organelles where the lipid kinases also reside. Phosphorylation of PI to phosphatidylinositol $(4,5)$ bisphosphate $\left(\mathrm{PI}(4,5) \mathrm{P}_{2}\right)$ at the plasma membrane and to phosphatidylinositol 4-phosphate (PI4P) at the Golgi are key events in lipid signalling and Golgi function respectively. Here we review a family of proteins, phosphatidylinositol transfer proteins (PITPs), that can mobilise PI from the ER to provide the substrate to the resident kinases for phosphorylation. Recent studies identify specific and overlapping functions for the three soluble PITPs (PITP $\alpha$, PITP $\beta$ and PITPNC1) in phospholipase C signalling, neuronal function, membrane trafficking, viral replication and in cancer metastases.
\end{abstract}

\section{Introduction}

Phosphoinositides, phosphorylated derivatives of phosphatidylinositol (PI), are key regulators of diverse cellular processes. These include plasma membrane receptor signalling through phospholipase Cs and PI 3-kinases, membrane trafficking, lipid exchange, ion channel regulation and cytoskeletal remodelling. The 3,4 , and 5 positions of the inositol ring of PI are accessible for phosphorylation individually and in combination, resulting in seven distinct phosphoinositides with phosphatidylinositol 4-phosphate (PI4P) and phosphatidylinositol $(4,5)$ bisphosphate $\left(\mathrm{PI}(4,5) \mathrm{P}_{2}\right)$ being the most abundant species. The 3-phosphorylated species (PI3P PI $(3,4) \mathrm{P}_{2}, \mathrm{PI}(3,5) \mathrm{P}_{2}$ and $\left.\mathrm{PI}(3,4,5) \mathrm{P}_{3}\right)$, and PI5P are present at substantially lower amounts in comparison. Phosphoinositides are the products of lipid kinases and phosphatases that are present in many subcellular compartments including the Golgi, endosomes and the plasma membrane. However, as the site of PI synthesis is the ER, PI delivery to other membrane compartments is required [1]. The hydrophobic nature of lipids in general means they require specific mechanisms for their distribution to target membranes. Whilst vesicular transport can deliver bulk lipids to target membranes, the more specific mechanism for delivery of specific lipids is accomplished by lipid transport proteins (LTPs). Many different families of LTPs have now been characterised and are widely distributed both intracellularly and extracellularly $[2,3]$. LTPs are characterised by a lipid binding domain that can encapsulate a single lipid monomer; the domain can exist as a single-domain soluble protein or be part of a multi-domain protein. A major development in the LTP field is the observation that many LTPs deliver lipids at membrane contact sites [4]. For multi-domain proteins, the lipid transfer domain co-exists with membrane-targeting domains that can facilitate the formation of membrane contact sites. Some LTPs may also associate with pre-existing membrane contact sites formed by other tethering factors. Whether single domain LTPs also function at membrane contact sites is not known, and if they do, it remains a major challenge to identify how they are recruited. Until now, LTPs were thought to bind and transfer a single lipid molecule but recent studies have identified proteins that contain a long hydrophobic groove that can bridge two membranes; the hydrophobic tails can thus slide through the groove between the two membranes. Both ATG2 and VPS13 proteins use this strategy to move lipids. The discovery and functions of ATG2 in autophagy and VPS13 proteins are reviewed elsewhere in this Special Issue $[5,6]$.

The family of LTPs, that can bind and transport PI are the phosphatidylinositol transfer proteins, PITPs (Fig. 1). The PITP family in mammalian cells comprises of five members, subdivided into two classes; Class I PITPs, PITP $\alpha$ and PITP $\beta$ which are single domain PITPs and three Class II PITPs which are subdivided into Class IIA and Class IIB. Class IIA PITPs are larger proteins containing additional domains that are reviewed elsewhere in this Special Issue [7]. The single Class IIB PITP, PITPNC1 contains a PITP domain with a C-terminal extension of 60 amino acids. This review will be focussed on the three soluble PITPs,

\footnotetext{
* Corresponding author.

E-mail address: s.cockcroft@ucl.ac.uk (S. Cockcroft).
} 
PITP $\alpha$, PITP $\beta$ and PITPNC1. Whilst the lipid binding and transfer activities are well-established for these PITPs, the cellular function of different members of the PITP family are only just beginning to be understood, facilitated by the use of model organisms. New studies reveal how the activity of PITP proteins is harnessed by different cell types to execute multiple functions including phototransduction, sensory behaviour, trafficking from the Golgi and also cell hijacking by viruses. In all these cases, phosphorylated PIs such as PI4P and PI $(4,5) \mathrm{P}_{2}$ are the primary mediators. In this review, how specificity of function could be derived based on the lipid-binding properties of the different PITP family members will be discussed.

\subsection{Introduction to PITPs}

The first PITP was identified due to its ability to bind and transfer phosphatidylinositol (PI), hence its name [8]. It was purified from bovine brain and the protein was found to consist of two forms in equal amounts, one form was bound to PI and the second form was bound to phosphatidylcholine (PC). A second PITP was subsequently identified by complementation of a temperature-sensitive Sec14 mutant in Saccharomyces cerevisiae [9]. It was simultaneously purified from bovine brain and designated as PITP $\beta$ with the original PITP referred to as PITP $\alpha$ [10].
PITPs are found not only in multicellular organisms but also in single cell organisms such as Giardia, E. cuniculi, Plasmodium falciparum, Dictyostelium and red algae, indicating their ancient origins [11,12]. PITPs belong to the larger StART family of proteins. However, the absence of PITP proteins in some well-studied model organisms including Saccharomyces cerevisiae, Schizosaccharomyces pombe and Arabidopsis thaliana is noteworthy. These organisms do contain PI transfer activity but this is due to another Class of proteins, the Sec14 family of proteins and are referred to as Sec14-like PITPs (See review by Griac et al. [13] in this Special Issue). PITPs of the StART family and Sec14-like PITPs have no sequence or structural homology [14]. Although members of both families can bind PI in a hydrophobic pocket, the orientation of the bound lipid is different. In PITPs, the headgroup of PI is buried within the pocket with the acyl chains pointing outwards, whilst in Sec14p, the tails are buried and the lipid headgroup is accessible [14]. It is of note that Sec14-like PITPs and StART-like PITPs can be simultaneously present in a simple organism such as Dictyostelium discoideum suggesting that the fundamental lipid transfer function of these proteins is specifically harnessed in different biological processes [15].

The StART family belongs to a large superfamily of proteins with a Bet v1 fold, an ancient versatile scaffold for binding hydrophobic ligands [16]. The distinctive arrangement of the secondary structure elements of

\section{Mammals}

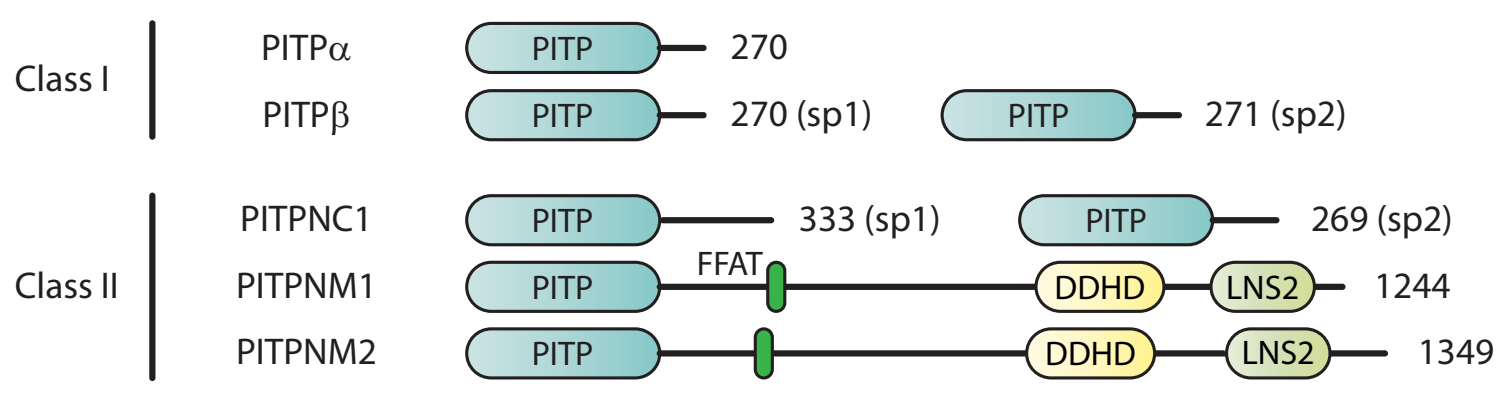

\section{Zebrafish}

Class I $\mid \begin{gathered}\text { Pitp } \alpha \\ \text { Pitp } \beta \\ \text { Pitp } \beta \text {-like }\end{gathered}$

Class II $\mid \begin{array}{r}\text { Pitpnc1a } \\ \text { Pitpnc1b } \\ \text { Pitpnm2 }\end{array}$

Drosophila
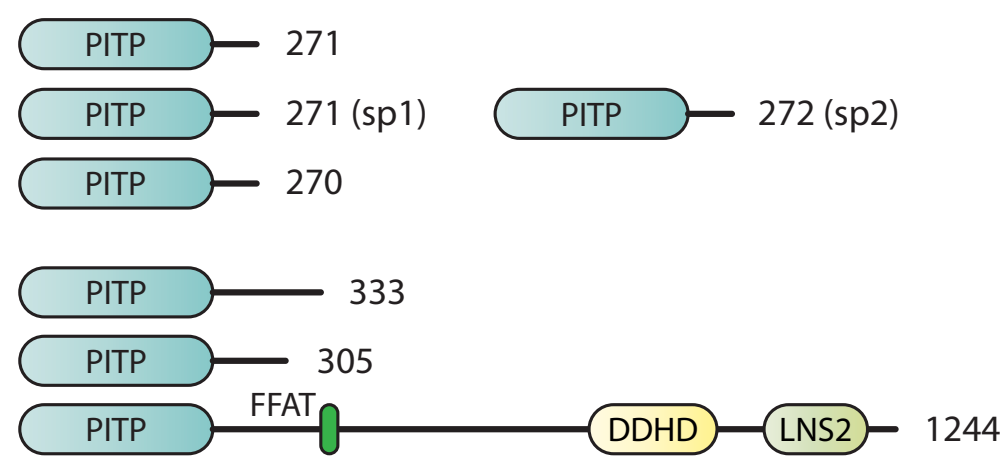
$\alpha$-helices and $\beta$-sheets gives rise to a three-dimensional structure consisting of a hydrophobic cavity, which is open to the exterior. Proteins with the Bet v1 fold are found among all kingdoms and diversified into numerous families with low sequence similarity but with a common fold during evolution. A large number of proteins make use of this scaffold to bind diverse hydrophobic ligands including phospholipids, ceramide and cholesterol. Both the PITP domain-containing proteins and the StART domain-containing proteins are included in this superfamily [17]. StART family proteins includes CERT that binds ceramide, StaR and MLN64 that binds cholesterol and PCTP that binds PC. More recently, the GRAM domain containing proteins, GramD1a, GramD1b and GramD1c were identified as proteins with a StART domain that binds cholesterol $[17,18]$. To date only the PITP family of this superfamily with a Bet v1 fold has been shown to bind PI.

The five proteins with a PITP domain present in the human genome are subdivided into two Classes based on two parameters; amino acid sequence and their lipid binding properties [19-21]. Class I PITPs, PITP $\alpha$ and PITP $\beta$, bind and transfer PI and PC whilst Class II PITPs that comprise of PITPNC1, PITPNM1 and PITPNM2 bind and transfer PI and PA. PITPNM proteins are also known as Nir proteins, a nomenclature based on their binding to the N-terminal domain of protein kinase PYK2 (PYK2 N-terminal domain-interacting receptors) [22]. The PITPNM nomenclature will be used here. PITPNM proteins are the mammalian counterparts of $\operatorname{RdgB} \alpha$, first identified in Drosophila as a retinal degeneration mutant, and hence its name [23]. The RdgB nomenclature is also used to describe the PITP family. Thus, PITPNC1 is also referred to as $\operatorname{RdgB} \beta$ [24-26].

Although the PITP family comprises of five members in mammals, the presence of splice variants increases their number. PITP $\alpha$ and PITP $\beta$ are small compact soluble proteins of $\sim 270$ amino acids and share $77 \%$ sequence identity and 95\% sequence similarity (Fig. 1). In humans, PITP $\alpha$ and PITP $\beta$ are localised on chromosome 17p13.3 and 22q12.1 respectively. The major difference between the two PITPs is the C-terminal 20 amino acids. In PITP $\alpha$ and $\beta$ the C-terminus ends with a helix (G helix in Fig. 2A) followed by an unstructured tail of 11 amino acids. Both proteins exhibit PI and PC binding and transfer activity. A notable difference between the two PITPs is that PITP $\beta$ has a higher affinity for binding to membranes and an increased transfer rate [27]. PITP $\beta$ is expressed as two splice variants which also differ in their extreme $\mathrm{C}$ termini [28]. The C-terminal 15 amino acids of the PITP $\beta$ splice variant 1 (sp1) are replaced by an alternative C-terminus of 16 amino acids in PITP $\beta$-sp2. The C-terminus of PITP $\beta$-sp1 is constitutively phosphorylated at Ser262, a residue that is lacking in PITP $\beta$-sp2. PITP $\beta$ is highly enriched in liver and the single gene gives rise to seven distinct protein species that differ in their phosphorylation status and the bound lipid state [28]. In vitro analysis of the lipid binding properties of the two splice variants suggest minor differences in their biochemical activity. Both variants bind PI and PC and are expressed in most cell types examined. In liver, similar amounts of the two splice variants are expressed at the protein level. PITP $\beta$ localises to the Golgi and it was suggested that Golgi localisation was dependent on phosphorylation of Ser262 [29]. However, subsequent studies indicated that both splice variants localise to the Golgi suggesting that phosphorylation is not essential $[28,30]$.

The Class IIB protein, PITPNC1 comprises a PITP domain followed by an unstructured C-terminal extension of 60 amino acids. In mammals there is a single gene that codes for two splice variants [25]. In humans, the PITPNC1 gene is on chromosome 17. The splice variant 1 is longer with 332 amino acids whilst PITPNC1-sp2 is 268 amino acids. The Cterminal extension in PITPNC1-sp1 contains two serine residues (Ser274 and Ser299) which form binding sites for 14-3-3 proteins when phosphorylated. The shorter splice variant does not interact with 14-3-3 proteins. Compared to Class I PITPs which are expressed in unicellular and multicellular organisms, expression of PITPNC1 is restricted. It is present in mammals, flies, amphibians and fish but not in C. elegans. In zebrafish, two separate genes encode for Pitpnc1 proteins, one on chromosome 3 (Pitpnc1a) and the other on chromosome 16 (Pitpnc1b). Pitpnc1a is 331 amino acids long with $81 \%$ identity and $90 \%$ similarity to PITPNC1-sp1. This protein also binds $14-3-3$ proteins. Pitpnc1b is shorter with 305 amino acids and is similar to PITPNC1-sp2 (Fig. 1). The complex expression patterns and isoform splicing of the PITP family across multiple organisms suggests that the proteins have evolved to play discrete roles in specific tissues. In order to understand these roles, it is important to think how the proteins perform their functions. Many studies have been carried out to elucidate the structure of PITPs and how they are able to bind and transfer lipid monomers.

\subsection{Structure of the PITP domain}

Crystal structures of the lipid-loaded and apo-structures of Class I PITPs as well as molecular dynamics simulations have begun to shed light on how PITPs interacts with membranes for lipid exchange [12,31-34]. X-ray crystal structures of three distinct forms of PITP $\alpha$ have been reported; PITP $\alpha$ bound to PC [12] or to PI [32], and one that is devoid of lipid [31]. In addition, the structure of PITP $\beta$ loaded with PC is also available [33]. The lipid-loaded structures of either PITP $\alpha$ or PITP $\beta$, regardless of the lipid-bound, are near identical; this excludes conformational changes in the structure regulated by the identity of the encapsulated lipid. The structure of PITP can be subdivided into four regions, the lipid binding core (coloured yellow), the regulatory region (coloured blue), the C-terminal G-helix ending with an unstructured extension (coloured red) and the lipid exchange loop (coloured green) (Fig. 2A). Fig. 2A is a modelled structure of PITPNC1 loaded with PI based on the crystal structures and looks remarkably similar to the structures of PITP $\alpha / \beta$. The $\beta$-strands form a large concave sheet flanked by two long $\alpha$-helices (coloured yellow in Fig. 2A), that form the lipid binding cavity. The hydrophobic cavity can be occupied by only a single phospholipid molecule. In the case of PITPNC1, it would be either PI or PA. Access to the cavity is obstructed by a 'lid' composed of the G-helix and C-terminal unstructured extension (coloured red in Fig. 2A). The headgroup of the bound lipid is accommodated deep inside the cavity whilst the $s n 1$ and $s n 2$ fatty acyl chains point towards the 'lid'. With the 'lid' closed, the phospholipid is completely enclosed within the protein [35]. Due to the location of the inositol ring which is buried deep within the lipid binding cavity, phosphorylation by lipid kinases is not physically possible. In the structure of the lipid-free form, the lipid exchange loop and the G-helix has swung outwards from the main structure, allowing passage of the phospholipid into or out of the cavity.

What controls the dynamics of the protein cycling between the 'open' and 'closed' is the interaction with a membrane [34,35]. Various regions of the PITP $\alpha$ molecule have been identified that participate in membrane association. This includes the lipid exchange loop, the G-helix and two tryptophan residues (WW203/204) located at the tip of the loop between a $\beta$-strand and $\alpha$-helix F) (see Fig. 2A). Mutation of these residues in PITP $\beta$ disrupts Golgi localisation, and in vitro, the lipid transfer activity of both PITP $\alpha$, PITP $\beta$ and the PITP domain of Drosophila RdgB $\alpha$ is reduced. [32,35,36]. Molecular dynamics simulations of PITP $\alpha$ suggest that the lipid exchange loop inserts into the bilayer upon membrane binding [34]. The most likely amino acid within this loop to have membrane interaction properties is Phe72, however, mutation of this residue to alanine has no impact on lipid binding, lipid transfer or its ability to support phospholipase C signalling in permeabilised cells [32].

Class I and Class II PITPs show differences in their lipid binding characteristics. Both Class I and Class II PITPs can bind and transfer PI; the counter ligand is different. Class I can bind and transfer PC whilst Class II can bind and transfer PA. For PI binding and transfer, four amino acid residues, Thr59, Lys61, Glu86 and Asn90 located on two of the $\beta$-strands provide the binding sites for the inositol ring. Mutations of any of those individual residues leads to loss of PI binding but not that of PC in Class I PITPs [32,35,37]. These residues are conserved in the majority of PITP sequences available in the databases, a hallmark that is predictive of PI binding. For PC binding, Cys95 and Phe225 are important. 


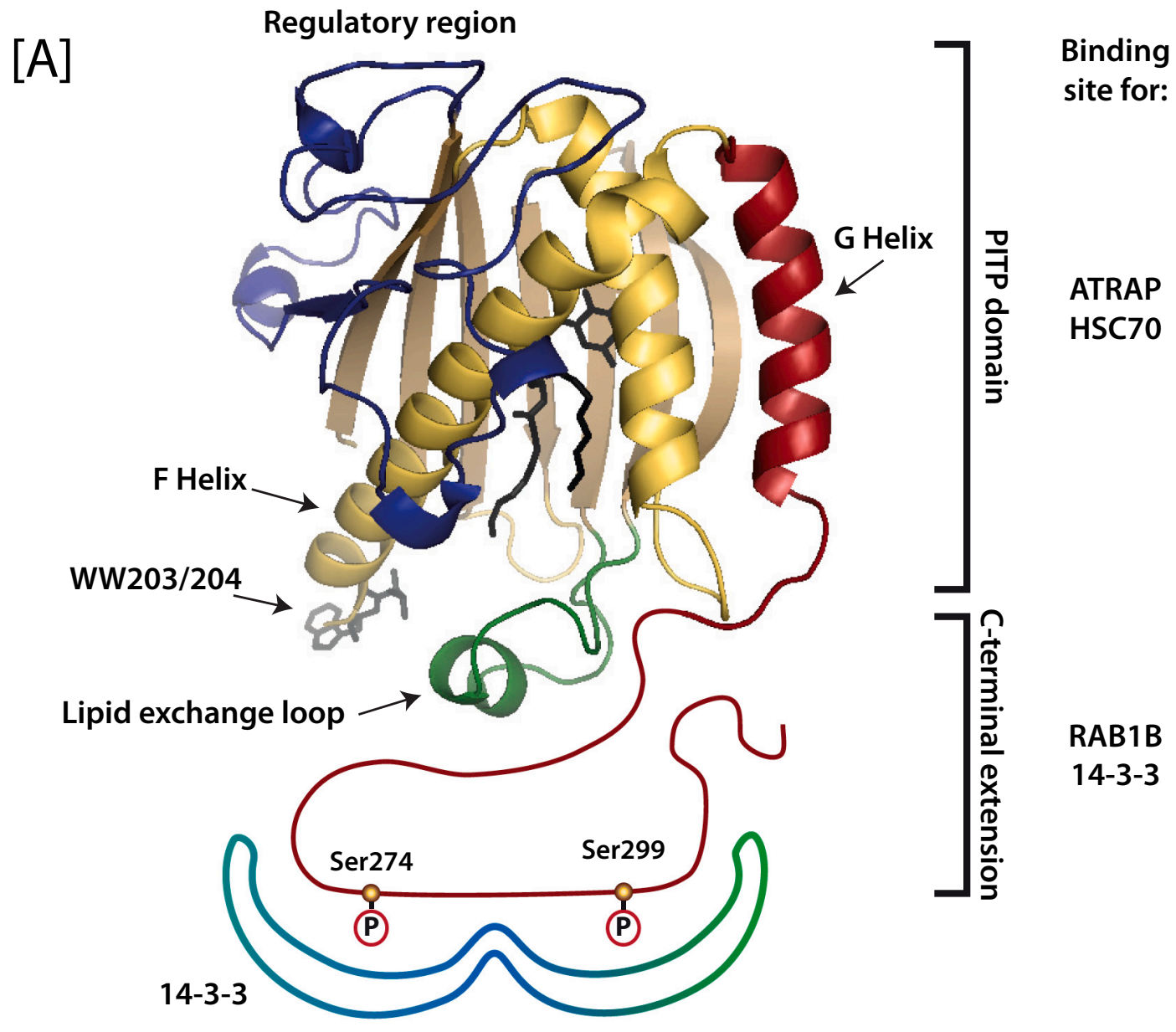

$[B]$
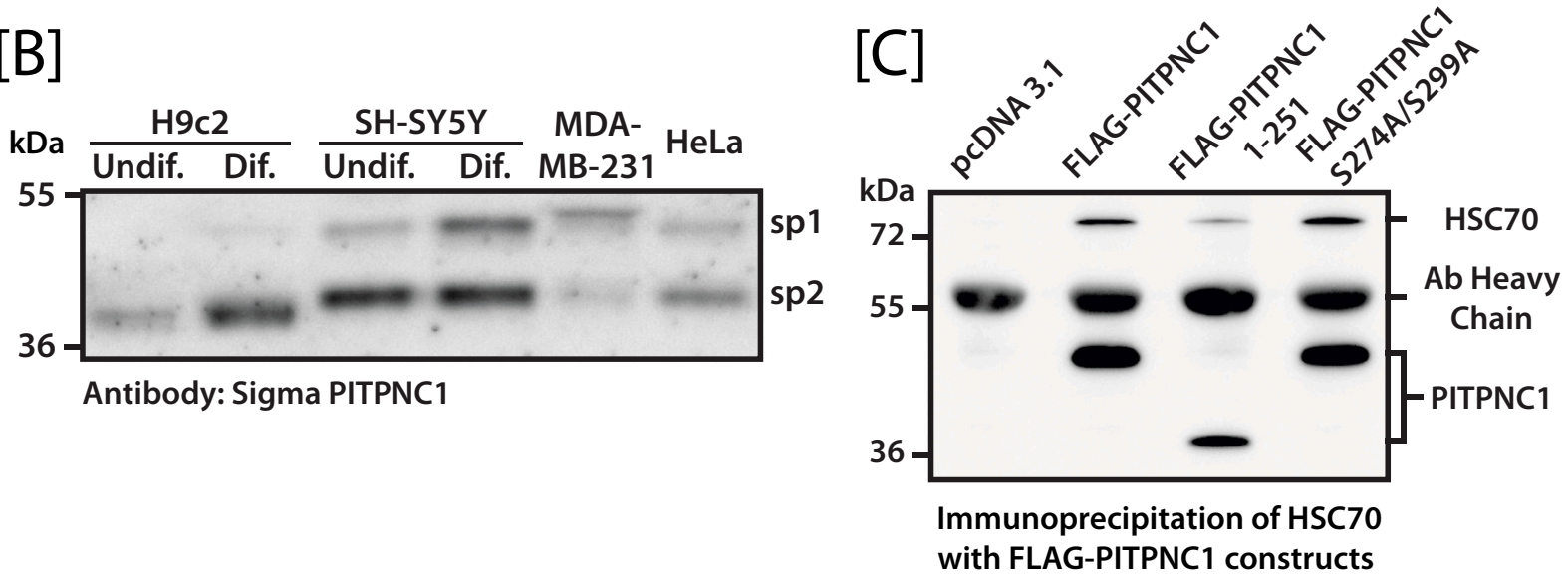

Fig. 2. Binding partners of PITPNC1. [A] The modelled structure of PITPNC1-sp1 bound to a 14-3-3 dimer. The lipid in the binding pocket is phosphatidylinositol. The phosphorylation sites, Ser274 and Ser299 which form the binding site for 14-3-3 are indicated. The PITP domain of PITPNC1 was modelled on the template with the PDB code 1T27 using MODELLER (adapted from [26]). The C-terminal extension coloured red is hypothetical. The putative binding sites for RAB1B, HSP70 and ATRAP are indicated. HSP70 and ATRAP bind to the PITP domain whilst 14-3-3 and RAB1B binds to the C-terminal extension. [B] Expression of PITPNC1 splice variants in multiple cell-lines. PITPNC1-sp1 is the longer form at 333 amino acids which contains an extended C-terminus which is disordered. PITPNC1-sp2 is 269 amino acids has a shorter C-terminal extension and does not contain the residues for 14-3-3 binding. H9c2 cells and SH-SY5Y cells were differentiated with all-trans retinoic acid in tissue culture media containing 1\% fetal calf serum for eight and five days respectively. [C] PITPNC1 binds to HSC70. FLAG-tagged PITPNC1 wild type, a truncated mutant containing residues 1-251 and a mutant with the 14-3-3 binding sites disrupted were transfected into COS-7 cells. All three constructs bind to HSC70. 
These residues are conserved in Class I but not Class II PITPs, reinforcing their inability to transfer PC [11]. Cys95 is located in the lipid binding cavity and mutation to either threonine or alanine results in loss of PC binding and transfer [37]. In Class II PITPs, this residue is replaced with threonine. Phe225 is not entirely conserved between human and rodents although among species, the amino acid sequences of PITP $\alpha$ and PITP $\beta$ are highly conserved (98-99\% identity). Whilst Phe225 is conserved in human PITP $\alpha$ and $\beta$, and in PITP $\alpha$ in rodents, PITP $\beta$ in rodents has a leucine in that position. PC transfer activity is increased when the leucine is mutated to phenylalanine, confirming the importance of this residue for PC transfer. Another feature are four amino acid residues (Gln22, Thr97, Thr114, and Lys195) that are conserved in the majority of PITPs; these residues are important for binding the phosphodiester moiety of either PI or PC.

Residues involved in PA binding remain to be identified in Class II PITPs. The best characterised PA transfer protein is a complex of PRELID1-TRIAP1 which transfers PA from the outer mitochondrial membrane to the inner mitochondrial membrane. Despite no significant sequence homology, the architecture of the PRELID1 domain is reminiscent of the PITP structure, an internal lipid binding pocket surrounded by a $\beta$-sheet and $\alpha$-helices $[38,39]$. The TRIAP1 domain is helical and is the equivalent of the G-helix in PITPs. Residues important for PA transfer has been identified based on structural analysis and also unbiased genetic approaches in yeast. However, lack of sequence homology between these proteins does not provide any clues about specific amino acid residues required for PA binding in PITPs. Thus, for Class II PITPs, structures with PA bound are needed. This would allow further analysis of the importance of PA binding in phospholipase $\mathrm{C}$ signalling and in other functions that these proteins participate in.

\subsection{The primary function of the PITP domain is to maintain phosphoinositide levels}

The recurring theme in PITP protein biology is the ability to maintain phosphoinositide levels [40,41]. Initial studies undertaken in permeabilised cells provided the first clues [42-45]. Depletion of PITPs by permeabilization led to loss of phospholipase C signalling, PI3K signalling, exocytosis and biogenesis of secretory granules and vesicles from the Trans Golgi Network (TGN) [42-49]. From these early studies, a clear concept emerged. PITP proteins promote the synthesis of phosphorylated derivatives of PI including PI4P, PI(4,5) $\mathrm{P}_{2}$ and $\mathrm{PI}(3,4,5) \mathrm{P}_{3}$ (reviewed in [41,50]).

The precise mechanism by which PITPs maintain phosphoinositide levels remains a question. Lipid delivery together with a cofactor function has been proposed [51]. The regulatory mechanism(s) that determine how PITPs are involved in maintaining phosphoinositides levels in different membrane compartments is not known. To date binding partners for Class I PITPs have not been identified. Below we discuss how individual PITPs function in cells at the level of the organism. Studies undertaken in mice, flies, zebrafish and dogs all point to both specific and over-lapping functions of the different members of the PITP families. This is not surprising because phosphoinositides, particularly PI4P and $\mathrm{PI}(4,5) \mathrm{P}_{2}$, play critical roles in cell signalling, membrane trafficking, cytoskeletal dynamics and cytokinesis [52-55].

Phosphatidylinositol is a negatively charged phospholipid and is present in mammalian cells at around 5-8\% depending on cell type [1]. In a typical cell, $5 \%$ of the PI will be found as PI4P whilst another $5 \%$ will be present as $\mathrm{PI}(4,5) \mathrm{P}_{2}$. Consumption of $\mathrm{PI}(4,5) \mathrm{P}_{2}$ during phospholipase C signalling can deplete PI levels by as much as $30 \%$ during intensive signalling [56]. PI synthesis is confined to the ER whilst the lipid kinases, PI4KIII $\alpha$ and PI4P 5-kinases are localised at the plasma membrane; thus, substrate delivery by PITPs is critical to maintain cellular homeostasis. The level of PI at the plasma membrane is negligible and is dependent on delivery from other sources [57,58]. Recent studies suggest that the Golgi, enriched in PI4P, can also contribute to PI $(4,5) \mathrm{P}_{2}$ recovery during agonist stimulation [59]. However, how PI4P would be delivered to the plasma membrane was not elucidated. Regardless, PITPs would still be required to deliver PI from the ER to the Golgi. Early studies in rat liver identified the principle organelles that were enriched with PI 4-kinase activity. These were the Golgi and plasma membrane with some activity in the lysosomes [60]. The ER, where PI is synthesised, was devoid of activity as was mitochondria. Four PI 4-kinases divided into Type II and Type III have now been identified in the human genome that are structurally distinct [61,62]. They are expressed in most cells; PI4KIII $\beta$ localises at the Golgi compartment whilst PI4KIII localises to the plasma membrane. The Type II PI 4-kinases, PI4KII $\alpha$ and PI4KII $\beta$ are palmitoylated enzymes and are also found at the Golgi and at endosomes [61-64].

\section{Class I PITPs}

\subsection{PITP $\alpha$ and PITP $\beta$ - neuronal studies in mice and Drosophila}

PITP $\alpha$ and PITP $\beta$ are the best studied of mammalian PITPs. Both are ubiquitously expressed proteins with PITP $\alpha$ expression being higher compared to PITP $\beta$ in most tissues with the exception of liver, lung and neutrophils. The highest levels of PITP $\alpha$ is found in the brain and histochemical analysis in the adult mouse cerebellum shows strong expression in the molecular layer and the granule cell layer [65]. The molecular layer is rich in synaptic contacts due to the dendritic tree of Purkinje cells and the parallel fibres of the granule cell layer. The dendritic spines of the Purkinje cells receive synaptic input from the parallel fibre. (In Drosophila, the single Class I PITP is required for dendritic morphology; in the absence of PITP, a reduction in branching is observed [66].) PITP $\alpha$ is also strongly expressed in the hippocampus including CA3 cells [65]. In mice, an essential requirement for PITP $\alpha$ for nervous system development and function was identified in the spontaneously-occurring vibrator mutation, which causes neurodegeneration due to reduced expression of PITP $\alpha$ by nearly 80\% [67]. The mice show a progressive whole-body tremor and spinocerebellar neurodegeneration. Expression of PITP $\beta$ is unperturbed implying a specific function of PITP $\alpha$ or insufficient amounts of PITP proteins. Mice where the PITP $\alpha$ gene is knocked out die within days after birth. These mice show a more complex phenotype; spinocerebellar neurodegeneration, intestinal and hepatic steatosis and hypoglycaemia [68]. Interestingly, when fatty liver disease is induced in zebrafish using perfluorooctane sulfonate, a widely used chemical or a fatty liver mutant, trappc11 ${ }^{-1-}$ (transport protein particle complex 11), transcription of PITP $\alpha$ is increased [69]. This increase could be a response by the cells trying to counteract the insult. Graded deletion of PITP $\alpha$ indicates that the brain is most affected when PITP $\alpha$ is partially reduced whilst other phenotypes only occur after total reduction in PITP $\alpha$ [70]. The binding of PI to PITP $\alpha$ is essential as a mutant unable to bind PI exhibit the same phenotype as the PITP $\alpha$-null mice [70].

Previous studies to make Pitpnb-null mice or null embryonic stem cells were not successful, concluding that PITP $\beta$ was an essential protein [71]. A more recent study was successful in making Pitpnb-null mice; they appear to be normal and fertile [72]. Attempts to make mice that were null for both PITP genes was unsuccessful indicating lethality. Instead, both PITPs were deleted specifically from the dorsal forebrain. Mice are born but lack the dorsal forebrain revealing that Class I PITPs are required for the development of the mammalian neocortex [72]. To identify the cellular mechanism leading to this phenotype, both PITPs were deleted in neural stem cells and this resulted in loss of polarity. The stem cells in the developing neocortex are unique bipolar epithelial cells, extending an apical process to the ventricle and a basal process to the basal lamina. In these cells, the Golgi apparatus is confined to the apical process. In the PITP-null cells, the Golgi redistributes from the apical process to a perinuclear location with defects in radial polarity. These phenotypes could be rescued with wild-type PITP $\alpha$ but not a mutant PITP that could not bind and transfer PI. GOLPH3 (Golgi phosphoprotein 3) localises to the Golgi by binding to PI4P and its localisation was 
disrupted in the PITP-null cells. CERT and FAPP1 also depend on PI4P for their Golgi localisation. Although their localisation was not examined in the PITP-null cells, knockdown of either GOLPH3, CERT or FAPP1 also resulted in Golgi positioning and radial alignment defects. However, only the localisation of GOLPH3 and CERT was dependent on PI4P, that of FAPP1 was not. GOLPH3 recruits MYO18A to regulate Golgi shape and was also found to show Golgi positioning and radial alignment defects when knocked down in neural stem cells of mouse embryos. Thus, PITP $\alpha$ and PITP $\beta$ together maintain a Golgi pool of PI4P which is required for Golgi positioning in the neural stem cells.

A recent study in mice where both PITP $\alpha$ and PITP $\beta$ were ablated suggests that PITP is also required for the noncanonical planar cell polarity (ncPCP) pathway, the core of which comprises the Wnt-Frizzled/ VANGL/PCP network [73]. The membrane traffic of VANGL2 from the TGN to the plasma membrane is disrupted in the absence of both PITPs. During embryonic development, the mammalian cortex expands in a lateral dimension increasing the neocortical area whilst restricting expansion in the radial dimension so that neocortical thickness is restricted, a process known as convergent expansion. Neural stem cells are responsible for this development. During neurogenesis, the nuclei change positions along the apical-basal axis, a process called interkinetic nuclear migration (INM) during the cell cycle to promote convergent extension [74]. At G1, the nuclei undergo apical to basal migration and after completion of S-phase, the nuclei undergo basal to apical migration. Mitosis is confined to the ventricular surface, as the apical plasma membrane harbours the primary cilium that is nucleated by the centrosomes that also build the mitotic spindle [75-77]. INM plays a critical role in cell fate determination and is compromised in neural stem cells when both Class I PITPs are deleted. This results in a thickened neocortex and perturbed curvature of its ventricular surface. These phenotypes are recapitulated in mouse embryos individually ablated for VANGL2 and Celsr1 suggesting that the ncPCP signalling is somehow connected to PITPs. PITPs appear to be required for the membrane trafficking of VANGL2 from the TGN to the neural stem cell surface. GOLPH3 is not required for this process suggesting that different PI4P effectors may be involved. These results suggest that neural development requires PITP for membrane trafficking of specific cargo proteins. These results can be best understood if the function of the Class I PITPs was to maintain Golgi PI4P. Loss of GOLPH3, CERT, and FAPP1 all depend on PI4P and moreover, PI4P participates in vesicle biogenesis from the TGN. These new studies provide further support that the PITPs are important for maintaining phosphoinositide levels in the Golgi compartment.

The importance of PITP in neuronal function is further supported by studies in Drosophila neural stem cells or neuroblasts, where the single Class I PITP (also known as vib or gio) and the PI 4-kinase, PI4KIII $\alpha$ were found to govern neuroblast polarity by anchoring non-muscle myosin II [78]. Neuroblasts divide asymmetrically to generate a self-renewing neuroblast and a differentiating daughter cell. During asymmetric division, polarized distribution of proteins occurs and are asymmetrically segregated into daughter cells. In PITP mutants, neuroblast homeostasis was perturbed with loss of neuroblasts. In addition, PITP was required for the localisation of apical and basal proteins and their faithful segregation in dividing neuroblasts. For example, in PITP mutants, aPKC and Par6 were found to redistribute from the apical cortex to the cytoplasm.

The non-muscle myosin II regulatory light chain protein, Spaghettisquash (Sqh) regulates asymmetric localisation of basal cell fate determinants in dividing neuroblasts. In Sqh mutants, aPKC is also mislocalised similarly to that seen in PITP mutant cells. Normally, Sqh is partially cytoplasmic with some localisation at the cell cortex in interphase cells and during metaphase it localises uniformly to the cell cortex. In PITP mutant cells, Sqh is completely cytosolic at interphase and partially cytosolic at metaphase indicating that Sqh is targeted to the cortex via PITP. PITP is responsible for promoting the synthesis of PI4P, and localisation of PI4P at the plasma membrane is lost in PITP mutants.
Of the three PI 4-kinases expressed in Drosophila, PI4KIII $\alpha$ was required for both neuroblast homeostasis and for asymmetric division. It was found to co-localise with Sqh to the cell cortex. Thus, PITP recruits Sqh and a direct interaction between the two proteins was demonstrated. In addition, Sqh also binds PI4P. Surprisingly, when Sqh was mutated, PI4P localisation was disrupted. Thus, Sqh requires both PITP and PI4P for recruitment to the cell cortex and PI4KIII $\alpha$ provides the PI4P whilst PITP provides PI for conversion to PI4P. PITP ${ }^{\mathrm{T} 59 \mathrm{E}}$ mutant is a wellcharacterised mutant; it is defective in PI binding and transfer but not that of PC. The equivalent mutant in Drosophila is PITP $^{\text {T63E }}$ and this mutant was unable to localise Sqh to the cell cortex. This comprehensive analysis provides the first evidence of how the complex interplay between PITP, PI4PKIII $\alpha$, and PI4P contribute to the recruitment of Sqh. Both PITP and Sqh are required for plasma membrane production of PI4P.

These two studies illustrate how PITPs contribute to localised PI4P production $[78,79]$. In the mouse model, where both Class I PITPs were disrupted, polarity of neural stem cells is lost. This is due to loss of PI4P at the Golgi where it is required for the trafficking of VANGL2 from the TGN to the neural stem cell surface. In the Drosophila model, the single PITP is required for PI4P production at the plasma membrane for recruitment of the non-muscle myosin II (Sqh) that governs neuroblast polarity. In these two studies, PITPs provide PI to different PI4Ks at separate localisations; PI4P at the plasma membrane through PI4KIII $\alpha$ and at the Golgi through PI4KIII $\beta$. This rules out direct interactions between PITPs and PI4Ks.

\subsection{GOLPH3, RABs and PITPS}

Drosophila PITP is also required for cytokinesis in spermatocytes and larval neuroblasts $[80,81]$. In addition to PITP, the PI4KIII $\beta$ (Fwd), GOLPH3 and Rab11 are also required for cytokinesis [82-85]. GOLPH3 is a highly conserved protein and binds to PI4P with high affinity [86]. It is essential for contractile ring formation and Rab11 localisation to the cleavage furrow during cytokinesis [87]. GOLPH3 binds to Sqh, nonmuscle myosin II light chain required for actomyosin contraction at the cleavage site. PITP and the PI4KIII $\beta$ participate in PI4P synthesis and are also required for the actomyosin ring constriction and cleavage furrow ingression. For cleavage furrow ingression, Golgi-derived vesicles are required and mutations in PITP or PI4KIII $\beta$ results in the abnormal localisation of Golgi-derived vesicles at the cell equator. Failure of fusion of these Golgi-derived vesicles with the invaginating furrow is responsible for the defects in cytokinesis. The Golgi-derived vesicles are Rab11-positive and are responsible for PITP and PI4KIII $\beta$ recruitment. The vesicles are recruited to the cleavage furrow and this requires the exocyst complex [88]. Thus PITP, together with Rab11 and $\mathrm{PI} 4 \mathrm{KIII} \beta$, is essential during exocyst-dependent membrane addition during cytokinesis in spermatocytes $[81,84,88]$. The single Drosophila PITP can participate in PI4P generation using different PI 4-kinases at different locations. During cytokinesis, PITP provides PI to PI4KIII $\beta$ whilst during asymmetric division of neuroblasts, PITP collaborates with PI4KIII $\alpha$.

In mammalian cells, GOLPH3 is localised to the TGN. Several studies suggest that it regulates anterograde trafficking of vesicles from the TGN to the plasma membrane. Other studies suggest that GOLPH3 is required for recycling glycosylation enzymes from the TGN to the cis-Golgi in COP1-coated vesicles [89-92]. GOLPH3 is over-expressed in many cancers and it is proposed that GOLPH3 might contribute to cellular transformation by affecting glycosylation of key cancer-relevant glycoproteins $[90,93,94]$. GOLPH3 also regulates Golgi to plasma membrane trafficking and contributes to malignant secretory phenotypes. In this case, another PITP, PITPNC1 (which will be described later), recruits RAB1B to the Golgi which interacts with GOLPH3 to recruit MYO18A. MYO18A by connecting to actin filaments, is thought to stretch the Golgi to cause membrane curvature and allow vesicle budding from the Golgi $[86,95]$. PI4P at the Golgi is synthesised by PI4KIII $\beta$ and this kinase is 
also amplified in cancer [97]. Thus, the PITPNC1/RAB1B/GOLPH3/ MYO18A/F-actin module participates in anterograde traffic and in some cancers, allows malignant secretion to take place. Whether PITPNC1 is required for intra-Golgi retrograde traffic is not known, but it is interesting to note that PITP $\beta$ is required for COP1-mediated traffic from the Golgi to the ER at least in HeLa cells [37]. Mutational analysis indicates that PITP has to be competent for both PI and PC exchange and also competent to dock on membranes [37]. Studies in both Drosophila and mammalian cells suggest a collaboration between RABs, GOLPH3 and PITPs as a recurring theme. The importance of PI4P in the recruitment of GOLPH3 is well-established but the link between RABs and GOLPH3 remains to be validated.

\subsection{PITP $\alpha$ and Duchenne muscular dystrophy}

Duchenne muscular dystrophy (DMD), an X-linked degenerative muscle disorder, is caused by the absence of a functional dystrophin protein. Dystrophin is a rod-shaped protein that links the intracellular cytoskeleton network to an integral membrane glycoprotein at the sarcolemma to provide structural stability and a signalling anchor to the muscle fibres. Loss of dystrophin causes a gradual decline of muscle function due to muscle degeneration. Recent studies in a colony of golden retriever muscular dystrophy dogs have identified that animals with decreased expression of PITP $\alpha$ exhibit a very mild phenotype [98]. In contrast, dogs that exhibit a severe phenotype have higher levels of PITP $\alpha$ compared to normal dogs. In these dogs, decreased expression of PITP $\alpha$ was associated with increased phosphorylated Akt (pAkt) expression and decreased PTEN (phosphatase and tensin homolog) levels. Further studies in DMD-patient derived muscle cells and in dystrophin-deficient zebrafish supported these observations. PITP $\alpha$ downregulation in DMD patient-derived muscle cells enhanced the formation of mature myotubes and increased pAkt [98]. PITP $\alpha$ knockdown by injection of morpholino oligonucleotides in a dystrophindeficient zebrafish also increased pAkt, rescued the abnormal muscle phenotype, and improved long-term survival.

Using the zebrafish dystrophin-deficient model, an inhibitor of the phosphodiesterase 10A (PDE10A) was found to reduce the manifestation of the dystrophic muscle phenotype. Moreover, PDE10A inhibition in zebrafish and in DMD patient-derived myoblasts were also associated with reduction of PITP $\alpha$ expression [99]. Thus, these results indicate a reduction in PITP $\alpha$ as a protective genetic modifier in dystrophindeficient model systems and permits the escape of the dystrophic phenotype. Decreased PITP $\alpha$ expression was associated with decreased levels of the PTEN and with increased Akt-phosphorylation. These effects promote muscle growth and metabolism to counteract the pathologies associated with muscular dystrophy. Similar changes in PTEN and Akt regulation were also observed following shRNA-mediated knockdown of PITP $\alpha$ in human DMD myoblasts and upon PITP $\alpha$ knockdown in a dystrophin-deficient sapje mutant zebrafish DMD model.

How does loss of PITP $\alpha$ lead to a decrease in PTEN with increased pAkt? A plausible explanation could be the regulation of PTEN by PI $(4,5) \mathrm{P}_{2}$. PTEN binds to $\mathrm{PI}(4,5) \mathrm{P}_{2}$ allowing recruitment to the membrane for synthesis of PI(3,4,5) $\mathrm{P}_{3}[100-102]$. In the absence of PITP $\alpha$, depletion of a specific pool of $\mathrm{PI}(4,5) \mathrm{P}_{2}$ utilised by PTEN for its recruitment, would result in increased $\mathrm{PI}(3,4,5) \mathrm{P}_{3}$ and thus pAkt. Why does inhibition of PDE10A cause a decrease in PITP $\alpha$ ? PDE10A can hydrolyse both cAMP and cGMP. Cyclic nucleotides could regulate PITP $\alpha$ levels through transcriptional regulation and this link needs further investigation. Regardless, these are interesting developments that could result in novel approaches to ameliorate the phenotypes in Muscular Duchenne patients.

\subsection{A requirement for PITPs in megakaryocytes and platelet function}

Mice knockouts of Class I PITP $\alpha$ results in severe phenotypes and thus conditional knockouts in specific cell-types would provide a better understanding of PITP $\alpha$ function. Conditional knockout mice lacking PITP $\alpha$ or both PITP $\alpha$ and PITP $\beta$ reveal important roles for PITPs in megakaryocytes and in platelets. Megakaryotes are derived from pluripotent hematopoietic stem cells (HSCs) and are large cells of 100 $\mu \mathrm{m}$ that give rise to platelets. Platelets are small cells $(2-3 \mu \mathrm{m})$ with no nucleus and are important for haemostasis. Platelets and megakaryotes are characterised by the presence of $\alpha$-granules and dense granules that contain a variety of mediators. $\alpha$-Granules contain proteins essential for platelet adhesion during vascular repair such as fibrinogen and Von Willebrand Factor (VWF), P-selectin, and angiogenesis regulatory proteins. Dense granules function predominantly to recruit additional platelets to sites of vascular damage and secrete ADP and ATP. ADP is a weak agonist triggering platelet shape change, granule release and aggregation.

Megakaryotes lacking both Class I PITPs have a defect in $\alpha$-granule morphology and show increased secretion of its contents including VWF, thrombospondin 1 and TGF $\beta 1$ into the extracellular medium. TGF $\beta 1$ maintains HSC quiescence and this results in decreased haematopoiesis. These data indicate that PITPs have a role in membrane trafficking, and in particular, in the biogenesis of secretory $\alpha$-granules. No defects were observed in dense granules. This is not surprising as the biogenesis of $\alpha$-granules and dense granules are distinct. In megakaryocytes, the biogenesis of $\alpha$-granules is formed from the TGN and early endosomes which then mature in multivesicular bodies (MVBs) [103]. Thus, the proteins stored in $\alpha$-granule are acquired from both the ER-to-TGN biosynthetic pathway and from the endocytic pathway that brings in plasma proteins. The $\alpha$-granules and the MVBs from the double PITP knockout megakaryocytes are less electron-dense and it was suggested that the $\alpha$-granule constituents were misdirected towards constitutive secretion into the medium [104]. Knockout of the single PITP $\alpha$ resulted in a milder phenotype. At what step PITPs are required for $\alpha$-granule biogenesis remains to be determined. PI4P has several roles in transport from the TGN, suggesting that it is the prime candidate [55]. PI4P can participate in the recruitment of coat proteins for vesicle biogenesis. Thus, PITPs are likely to provide PI for phosphorylation by the organelle-resident PI 4-kinases. The TGN and endosomes are both endowed with different PI4Ks and would imply localised production of PI4P at these sites.

Conditional knockouts of PITP $\alpha$ from mouse platelets reveals a requirement in thrombin-stimulated phospholipase $\mathrm{C}$ signalling. A reduction in $\mathrm{IP}_{3}$ production, in cytosolic $\mathrm{Ca}^{2+}$ and in PI4P and PI $(4,5) \mathrm{P}_{2}$ levels suggest defects in substrate provision for phospholipase $\mathrm{C}$ activity upon thrombin addition [105]. Nonetheless, some functional responses such as aggregation and $\alpha$-granule release are near normal when platelets are stimulated with thrombin. Although the major physiological function of platelets is in haemostasis, they can also contribute to tumour metastasis [106-108]. Thus, mice, with PITP $\alpha$ missing in their platelets, develop fewer lung metastases compared to normal mice when challenged with tumour cells. Normal platelets adhere to tumour cells forming a shroud and this protects the tumour cells from detection by immune cells. The tumour cells induce deposition of fibrin and thrombin generation; both of these functions are defective in platelets null for PITP $\alpha$. Thus, mice lacking PITP $\alpha$ in their platelets are protected from metastasis [105]. This very likely results from the failure of the platelets to expose phosphatidylserine (PS) by TMEM16F on the extracellular surface, a process that requires a rise in cytosol $\mathrm{Ca}^{2+}$ [109]. Exposed PS is required for thrombin generation and fibrin formation allowing tumour cells protection from the immune system.

\subsection{PITP $\beta$ participation in viral infection}

PITP $\beta$ has been implicated in viral replication of positive-strand RNA viruses of the Picornaviridae family. For viral RNA replication, the membranes of the ER and the Golgi are rearranged to generate a specific compartment, the replication organelle. PI4P is produced at the 
replication organelle by recruitment of PI4K via a Golgi-localised protein, ACBD3. PI4P production is commonly observed at viral replication sites for many other viruses [110-112]. Recent studies with the Aichi virus, which causes acute gastroenteritis in humans, hijacks OSBP to transport cholesterol from the ER to the replication organelle. This occurs at the ER-replication organelle membrane contact site. In addition to OSBP, VAPA/B, SAC1 and PITP $\beta$ are part of the cholesterol transport pathway. Depletion of any of these proteins results in loss of viral RNA replication. A complex comprising of viral proteins, ACBD3 and PI4KIII $\beta$ synthesises PI4P at the sites for viral RNA replication. OSBP is a transfer protein that uses the PI4P as a counter ligand to transfer cholesterol from the ER to the replication organelle [113,114]. At the ER, PI4P is dephosphorylated to PI by the PI4P phosphatase, SAC1. To regenerate PI4P at the replication organelle, PI is transferred by PITP $\beta$ (see Fig. 3). For Aichi virus replication, all the component proteins of the cholesterol transport machinery, OSBP, VAP, SAC1, and PITP $\beta$, are all essential host factors that are directly recruited to the RNA replication organelle $[115,116]$. Some viruses including SAR2-CoV2 use another PITP, the multi-domain PITPNM1 to accomplish the same function [117,118]. The strategy of combining multiple LTPs, OSBP and PITP $\beta$ or PITPNM1 for cholesterol transport is used by other organisms. In Toxoplasma gondii, instead of using multiple LTPs, a single protein of nearly 2000 amino acids that contains both the OSBP and the PITP domain can facilitate cholesterol transport [11,26,119].

\section{Class IIB PITPNC1}

The last of the mammalian PITPs to be identified was PITPNC1 (also known as $\operatorname{RdgB} \beta$ ), cloned from human fetal brain. PITPNC1 is longer than PITP $\alpha / \beta ; 333$ amino acids compared to 270 amino acids [24]. Like Class I PITPs, the $38 \mathrm{kDa}$ protein comprises the PITP domain followed by a C-terminal extension of 60 amino acids that is disordered. The gene is located on chromosome $17 \mathrm{q} 24.2$ with a pseudogene located on the long arm of chromosome 1q32.1. Sequence analysis allowed PITPNC1 to be classified with RdgB proteins as a Class IIB PITP [20]. PITPNC1 is ubiquitously expressed with enrichment in heart, muscle, kidney, liver and peripheral blood leukocytes.

The PITPNC1 gene is alternatively spliced giving rise to two splice variants. Splice variant 1 is the original protein at 333 amino acids; the shorter splice variant, sp2, is 269 amino acids. [25,120]. GFP-fusions of the two splice variants indicate that both proteins localise to the cytosol with the shorter variant also in the nucleus. Using in situ hybridization, the gene expression of the shorter form in the mouse brain was confined to the embryonic stage, whilst the longer form was widely expressed in the gray matter of pre- and postnatal brains. The dentate gyrus, the thalamus and the Purkinje cell layer in the cerebellum showed enrichment in the adult brain [120]. Interestingly, the gene expression of the two splice variants was also discrete in other mouse tissues; adult heart expressed both variants, the small intestine expressed the short form whilst the liver, kidney and testis only expressed the larger form.

PITPNC1 orthologues are restricted to jawed vertebrates (Gnathostomata) including, mammals, birds, turtles, lizards, amphibians and fish. In zebrafish. Pitpnc1 is expressed as two separate genes on different chromosomes instead of as splice variants. The pitpnc1a gene is present on chromosome 3 and encodes a protein of 331 amino acids, that shows $81 \%$ identity to human PITPNC1-sp1. The pitpnc1b gene is present on chromosome 16 and encodes a shorter protein of 305 amino acids which is the equivalent of the human PITPNC1-sp2 protein. The two proteins are differentially expressed: pitpnc1a is exclusively present in the brain with high expression in the forebrain, habenula and cerebellum. Pitpnc $1 \mathrm{~b}$ is excluded from the brain and is expressed in the pharyngeal arches, olfactory vesicles and the pronephric duct. The differential tissue distribution of the two proteins strongly indicate separate functions for these two proteins, likely to extend to the splice variants present in humans.

In cell-lines, both splice variants appear to be present with the expression of the smaller sp2 variant present at higher levels in most cases (Fig. 2B). Moreover, expression of both splice variants is increased following differentiation of the H92c cardiomyoblasts and SH-SY5Y neuronal cell-line using all-trans retinoic acid [121]. Interestingly, the breast cancer cell line, MDA-MB-231 is an exception which expresses a higher amount of $\mathrm{sp} 1$ compared to sp2. The observation that expression
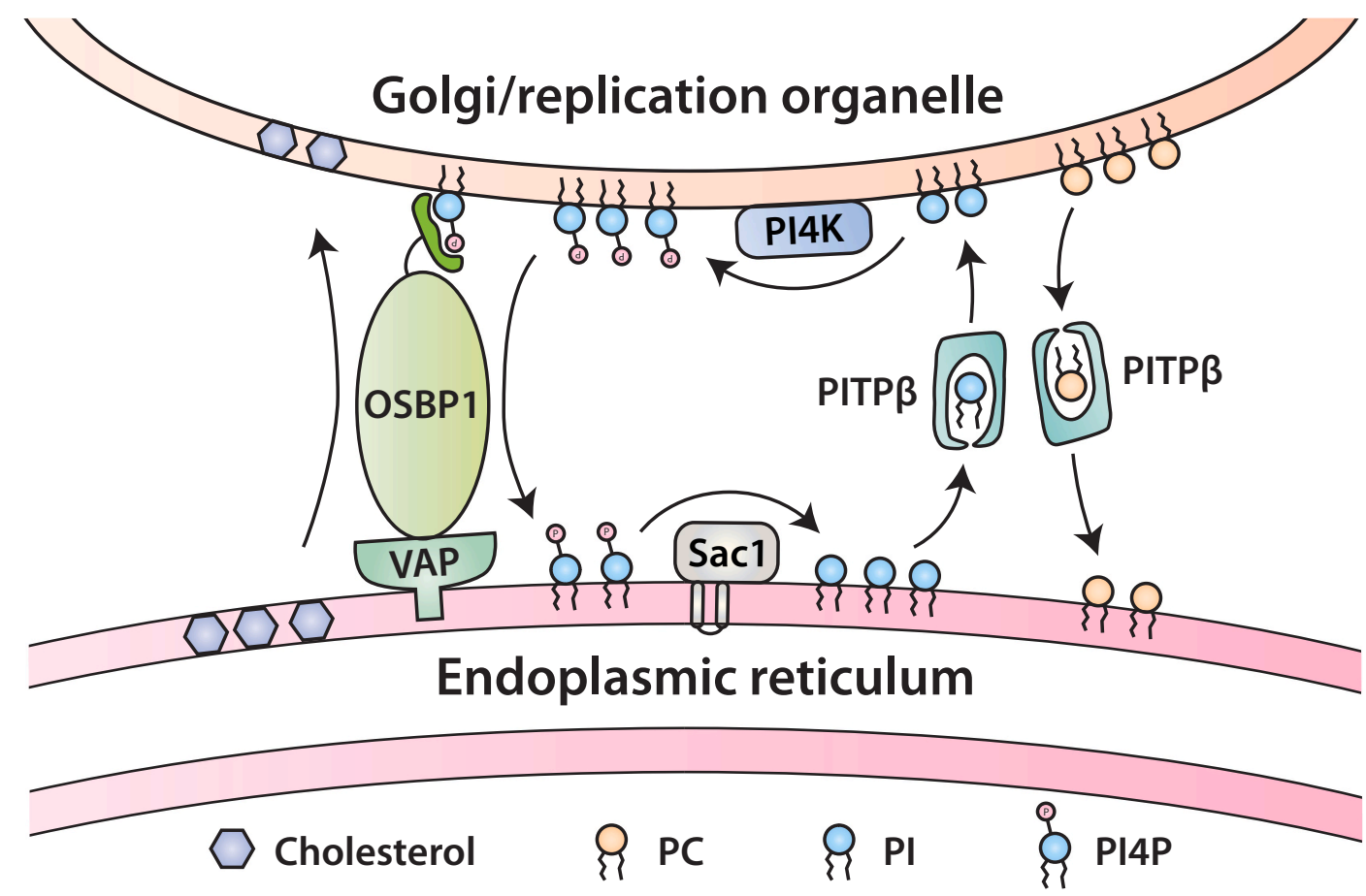

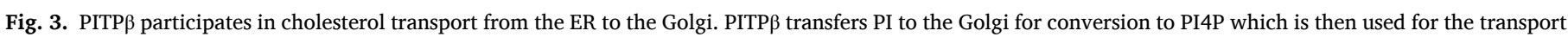

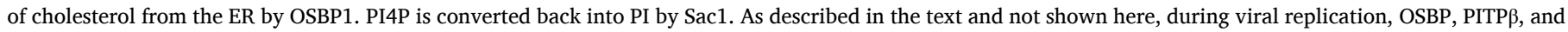
Sac1 are used to transport cholesterol to the replication organelle. 
of PITPNC1 increases following differentiation indicates that it is required for a cellular process in a mature cell such as a terminally differentiated cardiomyocytes and neurons.

\subsection{Binding partners for PITPNC1}

Several proteins have been identified that can bind PITPNC1. These are 14-3-3 proteins, ATRAP, HSC70 and RAB1B (Fig. 2A). The C-terminus of the longer splice variant, PITPNC1-sp1 contains two serine residues, (Ser274 and Ser299) which are phosphorylated creating a docking site for $14-3-3$ proteins $[25,26]$. Phosphorylation is very likely by protein kinase $C$ based on inhibitor studies [26]. Binding of 14-3-3 masks two PEST (Pro, Glu, Ser, Thr) sequences, that are present in proteins that show rapid turnover. Thus 14-3-3 binding protects the protein from degradation by the proteasome. However, compared to PITP $\beta$, which remains stable for up to $6 \mathrm{~h}$, wild type PITPNC1-sp1 has a half-life of about $4 \mathrm{~h}$, whilst the mutant protein that does not bind 14-3-3 is reduced to $2 \mathrm{~h}$. In addition to binding 14-3-3 proteins, PITPNC1 binds the transmembrane adapter protein, ATRAP. This interaction is dependent on protein kinase C activation. ATRAP can bind to both splice variants identifying the PITP domain as the site of interaction. Other proteins that binds to PITPNC1 are RAB1B [122] and HSC70 (heat shock cognate 70), a molecular chaperone (Blunsom and Cockcroft, unpublished) (Fig. 2C). HSC70 also binds to a truncated form of PITPNC1, one without the C-terminal region present. HSC70 is involved in facilitating protein maturation events and can transiently interact with nascent polypeptides in the process of synthesis. It is also involved in targeting proteins to the ubiquitin/proteasome machinery for ubiquitindependent degradation. This is in accord with the short half-life of the protein. RAB1B binding to PITPNC1 is not well-characterised but as binding of RAB1B to PITPNC1 is increased when a mutant that cannot bind 14-3-3 is used, this suggests that the binding site is in the C-terminal region, probably competing with 14-3-3 binding [122].

A study of the lipid binding and transfer properties of PITPNC1 reveals its unusual characteristics when compared to Class I or Class IIA PITPs. The PITP domain shares only 39\% identity with Class I and Class IIA proteins. The first notable feature of PITPNC1 is its poor in vitro PI transfer activity compared to both Class I and Class 2A PITPs. Secondly, it binds PA with very high affinity. Whilst PITP $\alpha$ can bind equal amount of PI and PC, PITPNC1 binds equal amounts of PI and PA. Considering that PC is generally present at $40 \%$ of total phospholipids whilst PA is present at less than 1\%, PA binding is of very high affinity. PITPNC1, when purified from E. coli, is also preloaded with PA and PG despite the low level of PA present. When PITPNC1 is exposed to permeabilised HL60 cells, the PA species bound to PITPNC1 are enriched for C16:0/ C16:1 and C16:1/C18:1, and increased binding to PA is observed at the expense of PI when cellular PA levels are increased. This is particularly the case when phospholipase $\mathrm{D}$ is activated. The fatty acid composition of the PA bound to PITPNC1 is characteristic of PC. Thus, PITPNC1 appears to respond to changes in PA levels as can occur during increased activity of phospholipase D or of diacylglycerol kinases. The significance of this is not known.

The biological function of Pitpnc1a has been examined in zebrafish. Pitpnc1a was knocked out using CRISPR and the adult fish developed normally and were fertile. The major phenotype observed in Pitpnc1anull zebrafish larvae is behavioural. The larvae lacking Pitpnc1a are hyperactive, with increased activity to touch, increased twitching and increase in waking activity. The increased neuronal activity was also evident biochemically; cFos expression was increased in many areas of the brain in the knockout animals. The cause of the hyperactivity is due to upregulation of IGF-1 receptor signalling. IGF-1 signalling is restrained by IGFBP2, and its secretion is likely to be impaired in cells lacking Pitpnc1a. This conclusion is supported by studies in cultured cells lines where knockdown of PITPNC1 result in a reduction of IGFBP2 secretion [123]. A recent study has identified that sleep disturbances can be affected by levels of IGF-1 by modulating the activity of orexin neurons [124]. This further supports that the PITPNC1/IGFBP2/IGF-1axis can regulate neuronal function and impacts on physiological behaviour.

Phosphatidic acid has emerged as a major regulator of cell function [125-127]. For example, studies in mice reveal that PLD1 is the major source of PA in the hippocampus and knockout mice show deficits in behaviour [128]. PA can also be made from DAG by diacylglycerol kinases (DGKs). Mammals express ten different enzymes that can use DAG with different fatty acid compositions [129,130]. DGKs are known to participate in neuronal function, cancer and Type II diabetes [129]. There is a strong possibility that PITPNC1 could be one of the effector proteins that senses PA to execute downstream function. As described below, PITPNC1 regulates malignant secretion from the Golgi resulting in metastasis.

\subsection{PITPNC1 and cancer}

Cellular lipid metabolism is altered in cancer. There are major changes in the amounts and the fatty acid profiles of the different lipids including PI and PA [131,132]. It is notable that breast cancer cell-lines have increased levels of PA, a lipid that is bound by PITPNC1 with high affinity [132,133]. In addition, the fatty acyl composition of PI is altered such that the acyl chain lengths are reduced [133]. Several studies indicate that PITPNC1 plays a pro-cancer role; it can promote tumour angiogenesis, metastasis, malignant secretion in breast cancer, omental metastasis of gastric cancer and in the development of radio resistance in colorectal cancer. Furthermore, PITPNC1 levels are increased in several different types of cancer (https://Cancer.Sanger.ac.uk/cosmic).

PITPNC1 was first recognised as a biomarker for human breast cancer metastasis [123]. A microRNA (miRNA)-126 that is silenced in a variety of human cancers, regulates the expression of IGFBP2, PITPNC1 and MERTK. (It is of note that miRNA-126 is also downregulated in paediatric B cells precursor acute lymphoblastic leukemia; however, its overexpression in MHH-CALL-3 leukemic cells does not reduce the expression levels of PITPNC1 [134].) The expression of IGFBP2 was dependent on PITPNC1. Subsequent work revealed that PITPNC1 was responsible for malignant secretion from the Golgi by facilitating the recruitment of RAB1B. RAB1B recruits GOLPH3 for driving vesicle formation for secretion of factors that drives invasion and angiogenesis. GOLPH3 is also highly expressed in many cancers. As discussed previously, many studies suggest that GOLPH3 participates in retrograde transport of glycosylation enzymes in COP1-coated vesicles from the TGN to the earlier Golgi compartments [92]. Thus, the mechanism by which PITPNC1 enhances secretion needs further work.

Two recent studies have examined the role of PITPNC1 in gastric cancer omental metastasis [135] and in radio-resistance in colorectal cancer [136]. Gastric cancer cells have elevated levels of PITPNC1 and this is due to the increased levels of cytokines, TNF $\alpha$ and IL6 secreted from adipocytes. Increased expression of PITPNC1 in gastric cancer cells results in increased activity of the transcription factors SREBP1 (Sterol regulatory element-binding protein 1) and $\operatorname{PPAR} \gamma$ (Peroxisome proliferator-activated receptor $\gamma$ ), regulating the expression of the fatty acid transporter, CD36 and carnitine O-palmitoyl transferase 1, which transports fatty acids into mitochondria. Effectively, PITPNC1 rewires the metabolism of the cancer cells to utilise fatty acids as fuel allowing the cancer cell to metastasise. What is of note is that the PITPNC1 responsible for this process appears to be the splice variant 2 , the shorter form, judging by the size of the protein. The splice variant, sp2, would not be expected to bind RAB1B suggesting that the two splice variants function in cancer metastasis by different mechanisms.

PITPNC1 is also implicated in resistance to radiotherapy used for therapy for colorectal cancer. Patients that show radio-resistance have a significantly higher level of PITPNC1 expression in rectal cancer tissue and produce significantly fewer ROS (reactive oxygen species). It is thought that PITPNC1 negatively regulates ROS production required for radiation-induced cell death. In support of this, PITPNC1 knockdown 
increased ROS production that could be reversed by a ROS scavenger [136].

In summary, PITPNC1 involvement in cancer is multifactorial. In addition to participating in secretion of malignant factors from the Golgi, in other cancers, the function is different.

\subsection{PITPNC1 and Type 2 diabetes}

GWAS studies had identified an expression-associated SNP in PITPNC1 (rs8866) as a risk factor for Type 2 diabetes [137]. Further support comes from another study where PITPNC1 was also identified as a risk locus using a genome-wide association study by proxy (GWAX) [138]. In GWAX, family members are used in the analysis. Observations that IGF-1 signalling is enhanced when PITPNC1 is deleted suggest that insulin signalling might be similarly affected by PITPNC1 [139].

\section{Perspectives and future directions}

PITPs are ubiquitous proteins and their biochemical activity of lipid transfer makes them well-suited for providing PI for conversion to PI4P and $\mathrm{PI}(4,5) \mathrm{P}_{2}$. The emerging picture is that PITPs function with different PI4K enzymes. PI4K enzymes are diverse and are localised in multiple organelles including the Golgi and the plasma membrane. Studies using model organisms indicate the diversity in the usage of phosphorylated PIs. This can occur both at the plasma membrane or the Golgi. At the plasma membrane, PITPs are required for $\mathrm{PI}(4,5) \mathrm{P}_{2}$ production for phospholipase C, and at the Golgi, PI4P is required for membrane traffic. Whilst the importance of PI binding and transfer is well-established in this process, it is not known whether PC metabolism is also affected by PITPs. Only one study has examined whether PC binding is important. In HeLa cells, COP1-dependent retrograde traffic was disrupted in PITP $\beta$ knockdown cells and rescue was only possible with a protein that retained both PI and PC transfer activity [37]. Further work is required to test this in other systems including in model organisms. An outstanding issue is the regulation of the PITP transfer activity. In cells, it is noted that the Class I PITPs are constantly sampling membranes undergoing an 'open' and 'closed' conformation [35]. Moreover, it is also noted that upon stimulation of cell surface receptors with appropriate agonists, PITP $\alpha$ and PITP $\beta$ can dynamically interact with PI and with PC [140]. This dynamic behaviour must be subject to regulatory controls and studies using new techniques such as hydrogen-deuterium exchange mass spectrometry may provide a deeper understanding of how these proteins interact with membranes.

Although PITP $\alpha$ and PITP $\beta$ are very similar is sequence, they appear to have both overlapping as well as specific functions. In both cases, the PITP activity is harnessed to participate in PI4P production. Depending on the compartment where PI4P is required, PI is provided by PITPs to be used by different PI 4-kinases. Thus, at the plasma membrane, PI4KIII $\alpha$ is responsible for PI4P synthesis whilst at the Golgi PI4KIII $\beta$ is responsible. This implies that PITPs do not directly interact with the PI4Ks but very likely deposit the lipid within the vicinity of the kinase. The interesting question is that of the composition of the membrane surrounding the area of PI4K localisation. Does the composition influence where PI is deposited in the membrane? Parameters that influence lipid transfer in vitro include membrane charge, fluidity, lipid composition and curvature $[141,142]$. How this operates in a cellular context is an unknown and this will require development of new tools to observe lipid transfer in real time in a living cell. However, studies in permeabilised cells and also in intact platelets indirectly indicate that delivery of PI does take place at the plasma membrane to account for increased hydrolysis of $\mathrm{PI}(4,5) \mathrm{P}_{2}$. Indeed, phospholipase $\mathrm{C}$ prefers to utilises the newly-synthesised $\mathrm{PI}(4,5) \mathrm{P}_{2}$ rather that the resident $\mathrm{PI}(4,5)$ $\mathrm{P}_{2}$ in the membrane $[51,105]$. This is not surprising as much of the PI $(4,5) \mathrm{P}_{2}$ may be unavailable, due to being bound to other proteins, including the actin cytoskeleton.

Not only is PITP $\alpha$ required for maintaining PLC activity in platelets stimulated by thrombin, it also has functions at the Golgi. In mice, PITP $\alpha$ and $\beta$ work with PI4KIII $\alpha$ in polarity establishment during neurogenesis [72]. In Drosophila, the single Class I PITP works together with Rab11 and PI4KIII $\beta$ during exocyst-dependent membrane addition during cytokinesis $[81,84,88]$ and also works together with PI4KIII $\alpha$ in polarity establishment during neurogenesis [78].

In contrast to Class I PITPs, the exact molecular mechanism behind the function of PITPNC1 remains enigmatic. Having said this, recent work in model organisms have begun to unlock the cellular function of PITPNC1. These studies have started to reveal some overlapping functions with Class I PITPs that are of interest to explore further. Broadly, PITPNC1 seems to regulate the morphology and function of the Golgi, and aberrant levels of PITPNC1 can alter levels of traffic through the secretory pathway. An example of this is seen in cancer, where increased levels of PITPNC1 lead to enhanced secretion of factors that can promote metastases of tumour cells [122]. Similarly, Pitpnc1a knockout studies in the zebrafish show that IGFBP2 secretion from neurons is reduced leading to over activation of the IGF-1 axis which manifests in behavioural phenotypes [139]. Although it appears that both Class I and Class II PITPs can regulate Golgi function, it is still unclear whether they achieve this by exactly the same biochemical mechanism.

Whilst PITPNC1 is classified as a lipid transfer protein, it shows poor transfer activity for both PI and PA compared to other PITPs. This observation suggests that the regulation of Golgi function occurs via a different mechanism to that achieved by PITP $\alpha$ and PITP $\beta$. One thing to note is that PITPNC1 is able to bind PA with very high affinity and this suggests that PITPNC1 is likely to participate in some aspect of PA signalling. Several studies have shown that PA produced via phospholipase D maintains the structural integrity of the Golgi [143]. Additionally, inhibition of PA production alters the structure of the Golgi and inhibits secretion from neuroendocrine cells [143-148]. In particular, monosaturated PA which preferentially binds to PITPNC1, regulates a number of exocytotic events [148]. More work is required to ascertain if PITPNC1 is regulating these processes through binding and/or transfer of PI/PA. PITPNC1 has been shown to have links to various pathologies including cancer, behavioural abnormalities and diabetes. This makes PITPNC1 a good candidate for further analysis to expand our knowledge of the mechanisms that lead to certain pathologies. These potential studies could lead to new therapeutic avenues for some diseases that have current clinical unmet needs.

The Class I and Class II PITPs are able to bind distinct combinations of phospholipids. Class I PITPs can bind PI and PC and the Class II PITPs can bind PI and PA. The question remains what physiological processes are supported by these specific phospholipid binding profiles. Experiments should be carried out in tissue-relevant cell models, which aim to knock out PITPs and assess the phenotypes induced by these changes. Rescue experiments could then be carried out with specific mutant PITPs with altered lipid binding profiles to pinpoint exact structure/function relationships. One area of focus for these phenotypic assays should be the lipid composition, morphology and secretory function of the Golgi apparatus. This is because the function of this organelle seems to link most phenotypes observed when PITP function is perturbed. It will be important to choose cellular systems that show high expression of the PITPs as pathologies from perturbation of PITP function usually occurs in tissues whereby the expression levels of PITPs are high.

There are four key areas of research that are outstanding. Development of inhibitors that can inhibit specific lipid binding to PITPs, structure of PITPNC1 bound to PA and to 14-3-3 and analysis of the splice variants of PITP $\beta$ and of PITPNC1. The splice variants of PITPNC1 is particularly interesting as the zebrafish model organism expresses the two proteins from separate genes. Finally, do soluble PITPs function at membrane contact sites and if so, how are they recruited? In conclusion, there is much to be discovered. 


\section{CRediT authorship contribution statement}

This article was jointly written by Tim Ashlin, Nicholas Blunsom and Shamshad Cockcroft.

\section{Declaration of competing interest}

The authors declare no competing interests.

\section{Acknowledgements}

Some of the work described was supported by grants from BBSRC (BB/J005606/1) and BHF (FS/15/73/31672). I want to thank past members of my Laboratory, Kathryn Garner and Evelyn Gomez-Espinosa for their contribution to the work on phosphatidylinositol transfer proteins. S. Cockcroft, ORCHID ID: 0000-0002-5731-476X.

\section{References}

[1] N.J. Blunsom, S. Cockcroft, Phosphatidylinositol synthesis at the endoplasmic reticulum, Biochim. Biophys. Acta Mol. Cell Biol. Lipids 1865(1) (2020) 158471.

[2] A. Chiapparino, K. Maeda, D. Turei, J. Saez-Rodriguez, A.C. Gavin, The orchestra of lipid-transfer proteins at the crossroads between metabolism and signaling, Prog. Lipid Res. 61 (2016) 30-39.

[3] L.H. Wong, A.T. Gatta, T.P. Levine, Lipid transfer proteins: the lipid commute via shuttles, bridges and tubes, Nat. Rev. Mol. Cell Biol. 20 (2) (2019) 85-101.

[4] Y. Saheki, P. De Camilli, Endoplasmic reticulum-plasma membrane contact sites, Annu. Rev. Biochem. 86 (2017) 659-684.

[5] M. Leonzino, K.M. Reinisch, P. De Camilli, Insights into VPS13 proerties and function reveal a new mechanism of eukaryotic lipid transport, Biochim. Biophys. Acta Mol. Cell Biol. Lipids (2021).

[6] N.N. Noda, Atg2 and Atg9 intermembrane and interleaflet lipid transporters driving autophagy, Biochim. Biophys. Acta Mol. Cell Biol. Lipids (2021).

[7] P.B. Raghu, H. Krishnan, Emerging perspectives on multidomain phosphatidylinositol transfer proteins, BBA (2021).

[8] G.M. Helmkamp Jr., M.S. Harvey, K.W.A. Wirtz, L.L.M. van Deenen, Phospholipid exchange between membranes. Purification of bovine brain proteins that preferentially catalyze the transfer of phosphatidylinositol, J. Biol. Chem. 249 (1974) 6382-6389.

[9] S. Tanaka, J.-I. Nikawa, H. Imai, S. Yamashita, K. Hosaka, Molecular cloning of rat phosphatidylinositol synthase cDNA by functional complementation of the yeast Saccharomyces cerevisiae pis mutation, FEBS Lett. 393 (1996) 89-92.

[10] K.J. De Vries, A.A.J. Heinrichs, E. Cunningham, F. Brunink, J. Westerman, P. J. Somerharju, S. Cockcroft, K.W.A. Wirtz, G.T. Snoek, An isoform of the phosphatidylinositol transfer protein transfers sphingomyelin and is associated with the golgi system, Biochem. J. 310 (1995) 643-649.

[11] S. Cockcroft, K. Garner, Function of the phosphatidylinositol transfer protein gene family: is phosphatidylinositol transfer the mechanism of action? Crit. Rev. Biochem. Mol. Biol. 46 (2011) 89-117.

[12] M.D. Yoder, L.M. Thomas, J.M. Tremblay, R.L. Oliver, L.R. Yarbrough, G. M. Helmkamp Jr., Structure of a multifunctional protein. Mammalian phosphatidylinositol transfer protein complexed with phosphatidylcholine, J. Biol. Chem. 276 (12) (2001) 9246-9252.

[13] R. Holic, D. Stastny, P. Griac, Sec14 family of lipid transfer proteins in yeast, Biochim. Biophys. Acta Mol. Cell Biol. Lipids (2021).

[14] A. Grabon, V.A. Bankaitis, M.I. McDermott, The interface between phosphatidylinositol transfer protein function and phosphoinositide signaling in higher eukaryotes, J. Lipid Res. 60 (2) (2019) 242-268.

[15] P. Swigart, R.H. Insall, A. Wilkins, S. Cockcroft, Purification and cloning of phosphatidylinositol transfer proteins from Dictyostelium discoideum: homologues of both mammalian PITPs and S. cerevisiae Sec14p are found in the same cell, Biochem. J. 347 (2000) 837-843.

[16] C. Radauer, P. Lackner, H. Breiteneder, The Bet v 1 fold: an ancient, versatile scaffold for binding of large, hydrophobic ligands, BMC Evol. Biol. 8 (2008) 286

[17] M. Khafif, L. Cottret, C. Balague, S. Raffaele, Identification and phylogenetic analyses of VASt, an uncharacterized protein domain associated with lipidbinding domains in eukaryotes, BMC Bioinf. 15 (2014) 222.

[18] A.T. Gatta, L.H. Wong, Y.Y. Sere, D.M. Calderon-Norena, S. Cockcroft, A. Menon, T.P. Levine, A new family of StART domain proteins at membrane contact sites has a role in ER-PM sterol transport, eLife 4 (2015) e07253.

[19] J. Hsuan, S. Cockcroft, The PITP family of phosphatidylinositol transfer proteins, Genome Biol. 2(9) (2001) 3011.1-3011.8.

[20] V. Allen-Baume, B. Segui, S. Cockcroft, Current thoughts on the phosphatidylinositol transfer protein family, FEBS Lett. 531 (2002) 74-80.

[21] K. Garner, A.N. Hunt, G. Koster, P. Somerharju, E. Grover, M. Li, P. Raghu, R. Holic, S. Cockcroft, Phosphatidylinositol transfer protein, cytoplasmic 1 (PITPNC1) binds and transfers phosphatidic acid, J. Biol. Chem. 287 (2012) 32263-32276.
[22] S. Lev, J. Hernandez, R. Martinez, A. Chen, G. Plowman, J. Schlessinger, Identification of a novel family of targets of PYK2 related to Drosophila retinal degeneration B (rdgB) protein, Mol. Cell. Biol. 19 (3) (1999) 2278-2288.

[23] T.S. Vihtelic, D.R. Hyde, J.E. O'Tousa, Isolation and characterisation of the Drosophila retinal degeneration B (rdgB) gene, Genetics 127 (1991) 761-768.

[24] Y. Fullwood, M. dos Santos, J.J. Hsuan, Cloning and characterization of a novel human phosphatidylinositol transfer protein, $\operatorname{rgdB} \beta$, J. Biol. Chem. 274 (1999) 31553-31558.

[25] K. Garner, M. Li, N. Ugwuanya, S. Cockcroft, The phosphatidylinositol transfer protein, RdgB $\beta$ binds 14-3-3 via its unstructured C-terminus, whereas its lipid binding domain interacts with the integral membrane protein, ATRAP (angiotensin II type I receptor-associated protein), Biochem. J. 439 (2011) 97-111.

[26] S. Cockcroft, K. Garner, 14-3-3 protein and ATRAP bind to the soluble class IIB phosphatidylinositol transfer protein RdgBbeta at distinct sites, Biochem. Soc. Trans. 40 (2) (2012) 451-456.

[27] M. Baptist, C. Panagabko, S. Cockcroft, J. Atkinson, Ligand and membranebinding behavior of the phosphatidylinositol transfer proteins PITPalpha and PITPbeta, Biochem. Cell Biol. 94 (6) (2016) 528-533.

[28] C.P. Morgan, V. Allen-Baume, M. Radulovic, M. Li, A.J. Skippen, S. Cockcroft, Differential expression of a C-terminal splice variant of PITP $\beta$ lacking the constitutive-phosphorylated Ser262 that localises to the golgi compartment, Biochem. J. 398 (2006) 411-421.

[29] C.M. van Tiel, J. Westerman, M.A. Paasman, M.M. Hoebens, K.W. Wirtz, G. T. Snoek, The Golgi localization of phosphatidylinositol transfer protein beta requires the protein kinase C-dependent phosphorylation of serine 262 and is essential for maintaining plasma membrane sphingomyelin levels, J. Biol. Chem. 277 (25) (2002) 22447-22452.

[30] S.E. Phillips, K.E. Ile, M. Boukhelifa, R.P. Huijbregts, V.A. Bankaitis, Specific and nonspecific membrane-binding determinants cooperate in targeting phosphatidylinositol transfer protein beta-isoform to the mammalian trans-Golgi network, Mol. Biol. Cell 17 (6) (2006) 2498-2512.

[31] A. Schouten, B. Agianian, J. Westerman, J. Kroon, K.W. Wirtz, P. Gros, Structure of apo-phosphatidylinositol transfer protein alpha provides insight into membrane association, EMBO J. 21 (9) (2002) 2117-2121.

[32] S.J. Tilley, A. Skippen, J. Murray-Rust, P. Swigart, A. Stewart, C.P. Morgan, S. Cockcroft, N.Q. McDonald, Structure-function analysis of human phosphatidylinositol transfer protein alpha bound to phosphatidylinositol, Structure 12 (2004) 317-326.

[33] P.B. Vordtriede, C.N. Doan, J.M. Tremblay, G.M. Helmkamp Jr., M.D. Yoder, Structure of PITP $\beta$ in complex with phosphatidylcholine: comparison of structure and lipid transfer to other PITP isoforms, Biochemistry 44 (2005) 14760-14771.

[34] A. Grabon, A. Orlowski, A. Tripathi, J. Vuorio, M. Javanainen, T. Rog, M. Lonnfors, M.I. McDermott, G. Siebert, P. Somerharju, I. Vattulainen, V. A. Bankaitis, Dynamics and energetics of the mammalian phosphatidylinositol transfer protein phospholipid exchange cycle, J. Biol. Chem. 292 (35) (2017) $14438-14455$.

[35] S. Shadan, R. Holic, N. Carvou, P. Ee, M. Li, J. Murray-Rust, S. Cockcroft, Dynamics of lipid transfer by phosphatidylinositol transfer proteins in cells, Traffic 9 (2008) 1743-1756.

[36] S. Yadav, K. Garner, P. Georgiev, M. Li, E. Gomez-Espinosa, A. Panda, S. Mathre, H. Okkenhaug, S. Cockcroft, P. Raghu, RDGBalpha, a PtdIns-PtdOH transfer protein, regulates G-protein-coupled PtdIns $(4,5) \mathrm{P} 2$ signalling during Drosophila phototransduction, J. Cell Sci. 128 (17) (2015) 3330-3344.

[37] N. Carvou, R. Holic, M. Li, C. Futter, A. Skippen, S. Cockcroft, Phosphatidylinositol- and phosphatidylcholine-transfer activity of PITPbeta is essential for COPI-mediated retrograde transport from the Golgi to the endoplasmic reticulum, J. Cell Sci. 123 (Pt 8) (2010) 1262-1273.

[38] X. Miliara, J.A. Garnett, T. Tatsuta, F.A. Ali, H. Baldie, I. Perez-Dorado, P. Simpson, E. Yague, T. Langer, S. Matthews, Structural insight into the TRIAP1/ PRELI-like domain family of mitochondrial phospholipid transfer complexes, EMBO Rep. 16 (2015) 824-835.

[39] X. Miliara, T. Tatsuta, J.-L. Berry, S.L. Rouse, K. Solak, D.S. Chorev, D. Wu, C.V. Robinson, S. Matthews, T. Langer, Structural determinants of lipid specificity within Ups/PRELI lipid transfer proteins, Nat. Commun. 10(1) (2019) 1130.

[40] S. Cockcroft, Phosphatidylinositol transfer proteins: a requirement in signal transduction and vesicle traffic, BioEssays 20 (1998) 423-432.

[41] S. Cockcroft, Phosphatidylinositol transfer proteins couple lipid transport to phosphoinositide synthesis, Semin. Cell Dev. Biol. 12 (2) (2001) 183-191.

[42] G.M.H. Thomas, E. Cunningham, A. Fensome, A. Ball, N.F. Totty, O. Troung, J. J. Hsuan, S. Cockcroft, An essential role for phosphatidylinositol transfer protein in phospholipase C-mediated inositol lipid signalling, Cell 74 (1993) 919-928.

[43] J.C. Hay, T.F.J. Martin, Phosphatidylinositol transfer protein required for ATPdependent priming of $\mathrm{Ca}^{2+}$-activated secretion, Nature 366 (1993) 572-575.

[44] J.C. Hay, P.L. Fisette, G.H. Jenkins, K. Fukami, T. Takenawa, R.E. Anderson, T.F. J. Martin, ATP-dependent inositide phosphorylation required for $\mathrm{Ca}^{2+}$-activated secretion, Nature 374 (1995) 173-177.

[45] G. Kular, M. Loubtchenkov, P. Swigart, J. Whatmore, A. Ball, S. Cockcroft, R. Wetzker, Co-operation of phosphatidylinositol transfer protein with phosphoinositide 3-kinase(gamma) in the formylmethionyl-leucylphenylalaninedependent production of phosphatidylinositol 3,4,5 trisphosphate in human neutrophils, Biochem. J. 325 (1997) 299-301.

[46] M. Ohashi, K. Jan de Vries, R. Frank, G. Snoek, V. Bankaitis, K. Wirtz, W. B. Huttner, A role for phosphatidylinositol transfer protein in secretory vesicle formation, Nature 377 (1995) 544-547. 
[47] J.-P. Simon, T. Morimoto, V.A. Bankaitis, T.A. Gottlieb, I.E. Ivanov, M. Adesnik, D.D. Sabatini, An essential role for the phosphatidylinositol transfer protein in the scission of coatomer-coated vesicles from the trans-Golgi network, Proc. Natl. Acad. Sci. U. S. A. 95 (1998) 11181-11186.

[48] G.S. Kular, A. Chaudhary, G. Prestwich, P. Swigart, R. Wetzker, S. Cockcroft, Cooperation of phosphatidylinositol transfer protein with phosphoinositide 3-kinase $\gamma$ in vitro, Adv. Enzym. Regul. 42 (2002) 53-61.

[49] A. Fensome, E. Cunningham, S. Prosser, S.K. Tan, P. Swigart, G. Thomas, J. Hsuan, S. Cockcroft, ARF and PITP restore GTP $\gamma$ S-stimulated protein secretion from cytosol-depleted HL60 cells by promoting PIP $_{2}$ synthesis, Curr. Biol. 6 (1996) 730-738.

[50] S. Cockcroft, The diverse functions of phosphatidylinositol transfer proteins, Curr. Top. Microbiol. Immunol. 362 (2012) 185-208.

[51] E. Cunningham, G.M.H. Thomas, A. Ball, I. Hiles, S. Cockcroft, Phosphatidylinositol transfer protein dictates the rate of inositol trisphosphate production by promoting the synthesis of $\mathrm{PIP}_{2}$, Curr. Biol. 5 (1995) 775-783.

[52] G.R.V. Hammond, J.E. Burke, Novel roles of phosphoinositides in signaling, lipid transport, and disease, Curr. Opin. Cell Biol. 63 (2020) 57-67.

[53] G. Di Paolo, P. de Camilli, Phosphoinositides in cell regulation and membrane dynamics, Nature 443 (7112) (2006) 651-657.

[54] M. Katan, S. Cockcroft, Phosphatidylinositol $(4,5)$ bisphosphate: diverse functions at the plasma membrane, Essays Biochem. 64 (2020) 513-531.

[55] M.A. De Matteis, C. Wilson, G. D'Angelo, Phosphatidylinositol-4-phosphate: the Golgi and beyond, Bioessays 35 (7) (2013) 612-622.

[56] N.J. Blunsom, E. Gomez-Espinosa, T.G. Ashlin, S. Cockcroft, Sustained phospholipase $\mathrm{C}$ stimulation of $\mathrm{H} 9 \mathrm{c} 2$ cardiomyoblasts by vasopressin induces an increase in CDP-diacylglycerol synthase 1 (CDS1) through protein kinase C and cFos, Biochim. Biophys. Acta Mol. Cell Biol. Lipids 1864(7) (2019) 1072-1082.

[57] J.G. Pemberton, Y.J. Kim, J. Humpolickova, A. Eisenreichova, N. Sengupta, D. J. Toth, E. Boura, T. Balla, Defining the subcellular distribution and metabolic channeling of phosphatidylinositol, J. Cell Biol. 219 (3) (2020), e201906130.

[58] J.P. Zewe, A.M. Miller, S. Sangappa, R.C. Wills, B.D. Goulden, G.R.V. Hammond Probing the subcellular distribution of phosphatidylinositol reveals a surprising lack at the plasma membrane, J. Cell Biol. 219(3) (2020) e201906127.

[59] J. Myeong, L. de la Cruz, S.R. Jung, J.H. Yeon, B.C. Suh, D.S. Koh, B. Hille, Phosphatidylinositol 4,5-bisphosphate is regenerated by speeding of the PI 4-kinase pathway during long PLC activation, J. Gen. Physiol. 152 (12) (2020), e202012627.

[60] S. Cockcroft, J.A. Taylor, J.D. Judah, Subcellular localisation of inositol lipid kinases in rat liver, Biochim. Biophys. Acta 845 (1985) 163-170.

[61] T. Balla, Phosphoinositides: tiny lipids with giant impact on cell regulation, Physiol. Rev. 93 (3) (2013) 1019-1137.

[62] E. Boura, R. Nencka, Phosphatidylinositol 4-kinases: function, structure, and inhibition, Exp. Cell Res. 337 (2) (2015) 136-145.

[63] J.E. Burke, Structural basis for regulation of phosphoinositide kinases and their involvement in human disease, Mol. Cell 71 (5) (2018) 653-673.

[64] S. Minogue, M.G. Waugh, The phosphatidylinositol 4-kinases: don't call it a comeback, Subcell. Biochem. 58 (2012) 1-24.

[65] K.E. Cosker, S. Shadan, M. van Diepen, C. Morgan, M. Li, V. Allen-Baume, C. Hobbs, P. Doherty, S. Cockcroft, B.J. Eickholt, Regulation of PI3K signalling by the phosphatidylinositol transfer protein PITP\{alpha\} during axonal extension in hippocampal neurons, J. Cell Sci. 121 (2008) 796-803.

[66] Y.-H. Wang, Z.-Y. Ding, Y.-J. Cheng, C.-T. Chien, M.-L. Huang, An efficient screen for cell-intrinsic factors identifies the chaperonin CCT and multiple conserved mechanisms as mediating dendrite morphogenesis, Front. Cell. Neurosci. 14 (311) (2020) 577315.

[67] B.A. Hamilton, D.J. Smith, K.L. Mueller, A.W. Kerrebrock, R.T. Bronson, V.v. Berkel, M.J. Daly, L. Kroglyak, M.P. Reeve, J.L. Nernhauser, T.L. Hawkins, E.M. Rubin, E.S. Lander, The vibrator mutation causes neurogeneration via reduced expression of PITP $\alpha$ : positional complementation cloning and extragenic suppression., Neuron 18 (1997) 711-722.

[68] J.G. Alb Jr., J.D. Cortese, S.E. Phillips, R.L. Albin, T.R. Nagy, B.A. Hamilton, V. A. Bankaitis, Mice lacking phosphatidylinositol transfer protein alpha exhibit spinocerebellar degeneration, intestinal and hepatic steatosis, and hypoglycemia, J. Biol. Chem. 278 (2003) 33501-33518.

[69] W.K. Fai Tse, J.W. Li, A.C. Kwan Tse, T.F. Chan, J.C. Hin Ho, R.S. Sun Wu, C.K Chu Wong, K.P. Lai, Fatty liver disease induced by perfluorooctane sulfonate: novel insight from transcriptome analysis, Chemosphere 159 (2016) 166-177.

[70] J.G. Alb Jr., S.E. Phillips, L.R. Wilfley, B.D. Philpot, V.A. Bankaitis, The pathologies associated with functional titration of phosphatidylinositol transfer protein alpha activity in mice, J. Lipid Res. 48 (8) (2007) 1857-1872.

[71] J.G. Alb Jr., S.E. Phillips, K. Rostand, X. Cui, J. Pinxteren, L. Cotlin, T.G. Manning, S.J.D. York, J.F. Sontheimer, J.F. Collawn, V.A. Bankaitis, Genetic ablation of phosphatidylinositol transfer protein function in murine embryonic stem cells, Mol. Biol. Cell 13 (2002) 739-754.

[72] Z. Xie, S.K. Hur, L. Zhao, C.S. Abrams, V.A. Bankaitis, A golgi lipid signaling pathway controls apical golgi distribution and cell polarity during neurogenesis, Dev. Cell 44(6) (2018) 725-740 e4.

[73] Y. Yang, M. Mlodzik, Wnt-frizzled/planar cell polarity signaling: cellular orientation by facing the wind (Wnt), Annu. Rev. Cell Dev. Biol. 31 (2015) 623-646.

[74] A. Sutherland, R. Keller, A. Lesko, Convergent extension in mammalian morphogenesis, Semin. Cell Dev. Biol. 100 (2020) 199-211.

[75] E. Taverna, M. Götz, W.B. Huttner, The cell biology of neurogenesis: toward an understanding of the development and evolution of the neocortex, Annu. Rev. Cell Dev. Biol. 30 (1) (2014) 465-502.
[76] Y. Arai, E. Taverna, Neural progenitor cell polarity and cortical development, Front. Cell. Neurosci. 11 (2017) 384.

[77] E. Taverna, F. Mora-Bermudez, P.J. Strzyz, M. Florio, J. Icha, C. Haffner, C. Norden, M. Wilsch-Brauninger, W.B. Huttner, Non-canonical features of the Golgi apparatus in bipolar epithelial neural stem cells, Sci. Rep. 6 (2016) 21206.

[78] C.T. Koe, Y.S. Tan, M. Lönnfors, S.K. Hur, C.S.L. Low, Y. Zhang, P. Kanchanawong, V.A. Bankaitis, H. Wang, Vibrator and PI4KIII $\alpha$ govern neuroblast polarity by anchoring non-muscle myosin II, eLife 7 (2018) e33555.

[79] Z. Xie, V.A. Bankaitis, Neural stem cell interkinetic nuclear migration is controlled by phosphatidylinositol transfer protein/non-canonical planar cell polarity signaling, bioRxiv (2020) 2020.12.17.423231.

[80] M.K. Gatt, D.M. Glover, The Drosophila phosphatidylinositol transfer protein encoded by vibrator is essential to maintain cleavage-furrow ingression in cytokinesis, J. Cell Sci. 119 (2006) 2225-2235.

[81] M.G. Giansanti, S. Bonaccorsi, R. Kurek, R.M. Farkas, P. Dimitri, M.T. Fuller, P. Gatti, The Class I PITP Giotto is required for Drosophila cytokinesis, Curr. Biol. 16 (2006) 195-201.

[82] J.A. Brill, G.R. Hime, M. Scharer-Schuksz, M.T. Fuller, A phospholipid kinase regulates actin organization and intercellular bridge formation during germline cytokinesis, Development 127 (17) (2000) 3855-3864.

[83] G. Polevoy, H.C. Wei, R. Wong, Z. Szentpetery, Y.J. Kim, P. Goldbach, S K. Steinbach, T. Balla, J.A. Brill, Dual roles for the Drosophila PI 4-kinase four wheel drive in localizing Rab11 during cytokinesis, J. Cell Biol. 187 (6) (2009) 847-858.

[84] M.G. Giansanti, G. Belloni, M. Gatti, Rab11 is required for membrane trafficking and actomyosin ring constriction in meiotic cytokinesis of Drosophila males, Mol. Biol. Cell 18 (2007) 5034-5047.

[85] S. Sechi, A. Frappaolo, A. Karimpour-Ghahnavieh, R. Fraschini, M.G. Giansanti, A novel coordinated function of myosin II with GOLPH3 controls centralspindlin localization during cytokinesis in Drosophila, J. Cell Sci. 133 (21) (2020), jcs 252965 .

[86] H.C. Dippold, M.M. Ng, S.E. Farber-Katz, S.K. Lee, M.L. Kerr, M.C. Peterman, R. Sim, P.A. Wiharto, K.A. Galbraith, S. Madhavarapu, G.J. Fuchs, T. Meerloo, M. G. Farquhar, H. Zhou, S.J. Field, GOLPH3 bridges phosphatidylinositol-4phosphate and actomyosin to stretch and shape the golgi to promote budding, Cell 139 (2) (2009) 337-351.

[87] S. Sechi, G. Colotti, G. Belloni, V. Mattei, A. Frappaolo, G.D. Raffa, M.T. Fuller, M. G. Giansanti, GOLPH3 is essential for contractile ring formation and Rab11 localization to the cleavage site during cytokinesis in Drosophila melanogaster, PLoS Genet. 10(5) (2014) e1004305.

[88] M.G. Giansanti, T.E. Vanderleest, C.E. Jewett, S. Sechi, A. Frappaolo, L. Fabian, C. C. Robinett, J.A. Brill, D. Loerke, M.T. Fuller, J.T. Blankenship, Exocystdependent membrane addition is required for anaphase cell elongation and cytokinesis in Drosophila, PLoS Genet. 11(11) (2015) e1005632.

[89] W.L. Chang, C.W. Chang, Y.Y. Chang, H.H. Sung, M.D. Lin, S.C. Chang, C.H. Chen, C.W. Huang, K.S. Tung, T.B. Chou, The Drosophila GOLPH3 homolog regulates the biosynthesis of heparan sulfate proteoglycans by modulating the retrograde trafficking of exostosins, Development 140 (13) (2013) 2798-2807.

[90] R. Rizzo, D. Russo, K. Kurokawa, P. Sahu, B. Lombardi, D. Supino, M. Zhukovsky, A. Vocat, P. Pothukuchi, V. Kunnathully, L. Capolupo, G. Boncompain, C. Vitagliano, F.Z. Marino, G. Aquino, D. Montariello, P. Henklein, L. Mandrich, G. Botti, H. Clausen, U. Mandel, T. Yamaji, K. Hanada, A. Budillon, F. Perez, S. Parashuraman, Y.A. Hannun, A. Nakano, D. Corda, G. D’Angelo, A. Luini, The Golgi maturation-dependent glycoenzyme recycling controls glycosphingolipid biosynthesis and cell growth via GOLPH3. EMBO J. 40 (8) (2021), e107238.

[91] R. Rizzo, S. Parashuraman, G. D’Angelo, A. Luini, GOLPH3 and oncogenesis: what is the molecular link? Tissue Cell 49(2, Part A (2017) 170-174.

[92] L.G. Welch, S. Munro, A tale of short tails, through thick and thin: investigating the sorting mechanisms of Golgi enzymes, FEBS Lett. 593 (17) (2019) 2452-2465.

[93] A. Frappaolo, A. Karimpour-Ghahnavieh, S. Sechi, M.G. Giansanti, The close relationship between the golgi trafficking machinery and protein glycosylation, Cells 9 (12) (2020) 2652.

[94] T. Ohashi, S. Hegi, T. Fukunaga, A. Hosomi, K. Takegawa, Golgi localization of glycosyltransferases requires Gpp74p in Schizosaccharomyces pombe, Appl. Microbiol. Biotechnol. 104 (2020) 8897-8909.

[95] J. Rahajeng, R.S. Kuna, S.L. Makowski, T.T.T. Tran, M.D. Buschman, S. Li, N. Cheng, M.M. Ng, S.J. Field, Efficient golgi forward trafficking requires GOLPH3driven, PI4P-dependent membrane curvature, Dev. Cell 50(5) (2019) 573-585. e5.

[97] X. Tan, P. Banerjee, E.A. Pham, F.U.N. Rutaganira, K. Basu, N. Bota-Rabassedas, H.-F. Guo, C.L. Grzeskowiak, X. Liu, J. Yu, L. Shi, D.H. Peng, B.L. Rodriguez, J. Zhang, V. Zheng, D.Y. Duose, L.M. Solis, B. Mino, M.G. Raso, C. Behrens, I.I. Wistuba, K.L. Scott, M. Smith, K. Nguyen, G. Lam, I. Choong, A. Mazumdar, J.L. Hill, D.L. Gibbons, P.H. Brown, W.K. Russell, K. Shokat, C.J. Creighton, J.S. Glenn, J.M. Kurie, PI4KIII $\beta$ is a therapeutic target in chromosome 1q-amplified lung adenocarcinoma, Sci. Transl. Med. 12(527) (2020) eaax3772.

[98] N.M. Vieira, J.M. Spinazzola, M.S. Alexander, Y.B. Moreira, G. Kawahara, D. E. Gibbs, L.C. Mead, S. Verjovski-Almeida, M. Zatz, L.M. Kunkel, Repression of phosphatidylinositol transfer protein alpha ameliorates the pathology of Duchenne muscular dystrophy, Proc. Natl. Acad. Sci. U. S. A. 114 (23) (2017) 6080-6085.

[99] M.R. Lambert, J.M. Spinazzola, J.J. Widrick, A. Pakula, J.R. Conner, J.E. Chin, J. M. Owens, L.M. Kunkel, PDE10A inhibition reduces the manifestation of pathology in DMD zebrafish and represses the genetic modifier PITPNA, Mol. Ther. 29 (2020) 1086-1101. 
[100] J. Li, E.J. Tanhehco, B. Russell, Actin dynamics is rapidly regulated by the PTEN and PIP2 signaling pathways leading to myocyte hypertrophy, Am. J. Phys. Heart Circ. Phys. 307 (11) (2014) H1618-H1625.

[101] S.B. Johnston, R.T. Raines, Catalysis by the tumor-suppressor enzymes PTEN and PTEN-L, PLoS One 10(1) (2015) e0116898.

[102] R. Karn, I.A. Emerson, Molecular dynamic study on PTEN frameshift mutations in breast cancer provide $\mathrm{c} 2$ domain as a potential biomarker, J. Biomol. Struct. Dyn. (2020) 1-12.

[103] A. Sharda, R. Flaumenhaft, The life cycle of platelet granules [version 1; peer review: 2 approved], F1000Research 7 (236) (2018).

[104] M. Capitano, L. Zhao, S. Cooper, C. Thorsheim, A. Suzuki, X. Huang, A.L. Dent, M. S. Marks, C.S. Abrams, H.E. Broxmeyer, Phosphatidylinositol transfer proteins regulate megakaryocyte TGF-beta1 secretion and hematopoiesis in mice, Blood 132 (10) (2018) 1027-1038.

[105] L. Zhao, C.L. Thorsheim, A. Suzuki, T.J. Stalker, S.H. Min, L. Lian, G.D. Fairn, S. Cockcroft, A. Durham, S. Krishnaswamy, C.S. Abrams, Phosphatidylinositol transfer protein- $\alpha$ in platelets is inconsequential for thrombosis yet is utilized for tumor metastasis, Nat. Commun. 8 (1) (2017) 1216.

[106] M. Schlesinger, Role of platelets and platelet receptors in cancer metastasis, J. Hematol. Oncol. 11 (1) (2018) 125.

[107] L.J. Gay, B. Felding-Habermann, Contribution of platelets to tumour metastasis, Nat. Rev. Cancer 11 (2) (2011) 123-134.

[108] A.L. Palacios-Acedo, D. Mège, L. Crescence, F. Dignat-George, C. Dubois, L. Panicot-Dubois, Platelets, thrombo-inflammation, and cancer: collaborating with the enemy, Front. Immunol. 10 (2019) 1805.

[109] T. Fujii, A. Sakata, S. Nishimura, K. Eto, S. Nagata, TMEM16F is required for phosphatidylserine exposure and microparticle release in activated mouse platelets, Proc. Natl. Acad. Sci. 112 (41) (2015) 12800-12805.

[110] Y.S. Lim, S.B. Hwang, Hepatitis C virus NS5A protein interacts with phosphatidylinositol 4-kinase type IIIalpha and regulates viral propagation, J. Biol. Chem. 286 (13) (2011) 11290-11298.

[111] K.L. Berger, S.M. Kelly, T.X. Jordan, M.A. Tartell, G. Randall, Hepatitis C virus stimulates the phosphatidylinositol 4-kinase III alpha-dependent phosphatidylinositol 4-phosphate production that is essential for its replication, J. Virol. 85 (17) (2011) 8870-8883.

[112] K.L. Berger, J.D. Cooper, N.S. Heaton, R. Yoon, T.E. Oakland, T.X. Jordan, G. Mateu, A. Grakoui, G. Randall, Roles for endocytic trafficking and phosphatidylinositol 4-kinase III alpha in hepatitis C virus replication, Proc. Natl Acad. Sci. U. S. A. 106 (18) (2009) 7577-7582.

[113] B. Antonny, J. Bigay, B. Mesmin, The oxysterol-binding protein cycle: burning off PI(4)P to transport cholesterol, Annu. Rev. Biochem. 87(1) (2018) null.

[114] B. Mesmin, J. Bigay, J. Polidori, D. Jamecna, S. Lacas-Gervais, B. Antonny, Sterol transfer, PI4P consumption, and control of membrane lipid order by endogenou OSBP, EMBO J. 36 (21) (2017) 3156-3174.

[115] K. Ishikawa-Sasaki, S. Nagashima, K. Taniguchi, J. Sasaki, Model of OSBPmediated cholesterol supply to Aichi virus RNA replication sites involving protein-protein interactions among viral proteins, ACBD3, OSBP, VAP-A/B, and SAC1, J. Virol. 92(8) (2018) e01952-17.

[116] P.S. Roulin, M. Lotzerich, F. Torta, L.B. Tanner, F.J. van Kuppeveld, M.R. Wenk, U.F. Greber, Rhinovirus uses a phosphatidylinositol 4-phosphate/cholesterol counter-current for the formation of replication compartments at the ER-golgi interface, Cell Host Microbe 16 (5) (2014) 677-690.

[117] P. Samavarchi-Tehrani, H. Abdouni, J.D.R. Knight, A. Astori, R. Samson, Z.-Y. Lin, D.-K. Kim, J.J. Knapp, J. St-Germain, C.D. Go, B. Larsen, C.J. Wong, P. Cassonnet, C. Demeret, Y. Jacob, F.P. Roth, B. Raught, A.-C. Gingras, A SARS-CoV-2 - host proximity interactome, bioRxiv (2020) 2020.09.03.282103.

[118] H. Wang, A.W. Tai, Nir2 is an effector of VAPs necessary for efficient hepatitis C virus replication and phosphatidylinositol 4-phosphate enrichment at the viral replication organelle, J. Virol. 93(22) (2019) e00742-19.

[119] S. Cockcroft, P. Raghu, Phospholipid transport protein function at organelle contact sites, Curr. Opin. Cell Biol. 53 (2018) 52-60.

[120] N. Takano, Y. Owada, R. Suzuki, H. Sakagami, T. Shimosegawa, H. Kondo, Cloning and characterization of a novel variant (mM-rdgBbeta1) of mouse MrdgBs, mammalian homologs of Drosophila retinal degeneration B gene proteins, and its mRNA localization in mouse brain in comparison with other M-rdgBs, J. Neurochem. 84 (4) (2003) 829-839.

[121] N.J. Blunsom, E. Gomez-Espinosa, T.G. Ashlin, S. Cockcroft, Mitochondrial CDPdiacylglycerol synthase activity is due to the peripheral protein, TAMM41 and not due to the integral membrane protein, CDP-diacylglycerol synthase 1, Biochim. Biophys. Acta 1863(3) (2017) 284-298.

[122] N. Halberg, C.A. Sengelaub, K. Navrazhina, H. Molina, K. Uryu, S.F. Tavazoie, PITPNC1 recruits RAB1B to the golgi network to drive malignant secretion, Cancer Cell 29 (3) (2016) 339-353.

[123] K.J. Png, N. Halberg, M. Yoshida, S.F. Tavazoie, A microRNA regulon that mediates endothelial recruitment and metastasis by cancer cells, Nature 481 (7380) (2012) 190-194.

[124] J.A. Zegarra-Valdivia, J. Pignatelli, M.E. Fernandez de Sevilla, A.M. Fernandez, V. Munive, L. Martinez-Rachadell, A. Nunez, I. Torres Aleman, Insulin-like growth factor I modulates sleep through hypothalamic orexin neurons, FASEB J. 34 (12) (2020) 15975-15990.
[125] E. Tanguy, Q. Wang, H. Moine, N. Vitale, Phosphatidic acid: from pleiotropic functions to neuronal pathology, Front. Cell. Neurosci. 13 (2019) 2.

[126] R.C. Bruntz, C.W. Lindsley, H.A. Brown, Phospholipase D signaling pathways and phosphatidic acid as therapeutic targets in cancer, Pharmacol. Rev. 66 (4) (2014) 1033-1079.

[127] R. Thakur, A. Naik, A. Panda, P. Raghu, Regulation of membrane turnover by phosphatidic acid: cellular functions and disease implications, Front. Cell Dev. Biol. 7 (2019) 83.

[128] L. Santa-Marinha, I. Castanho, R.R. Silva, F.V. Bravo, A.M. Miranda, T. Meira, R. Morais-Ribeiro, F. Marques, Y. Xu, K. Point du Jour, M. Wenk, R.B. Chan, G. Di Paolo, V. Pinto, T.G. Oliveira, Phospholipase D1 ablation disrupts mouse longitudinal hippocampal axis organization and functioning, Cell Rep. 30(12) (2020) 4197-4208 e6.

[129] F. Sakane, F. Hoshino, C. Murakami, New era of diacylglycerol kinase, phosphatidic acid and phosphatidic acid-binding protein, Int. J. Mol. Sci. 21 (18) (2020) 6794

[130] F. Sakane, S. Mizuno, D. Takahashi, H. Sakai, Where do substrates of diacylglycerol kinases come from? Diacylglycerol kinases utilize diacylglycerol species supplied from phosphatidylinositol turnover-independent pathways, Adv Biol Regul 67 (2018) 101-108.

[131] M.L. Doria, Z. Cotrim, B. Macedo, C. Simoes, P. Domingues, L. Helguero, M. R. Domingues, Lipidomic approach to identify patterns in phospholipid profiles and define class differences in mammary epithelial and breast cancer cells, Breast Cancer Res. Treat. 133 (2) (2012) 635-648.

[132] M.L. Doria, C.Z. Cotrim, C. Simoes, B. Macedo, P. Domingues, M.R. Domingues, L. A. Helguero, Lipidomic analysis of phospholipids from human mammary epithelial and breast cancer cell lines, J. Cell. Physiol. 228 (2) (2013) 457-468.

[133] A. Naguib, G. Bencze, D.D. Engle, I.I. Chio, T. Herzka, K. Watrud, S. Bencze, D. A. Tuveson, D.J. Pappin, L.C. Trotman, p53 mutations change phosphatidylinositol acyl chain composition, Cell Rep. 10 (1) (2015) 8-19.

[134] F. Akbari Moqadam, J.M. Boer, E.A. Lange-Turenhout, R. Pieters, M.L. den Boer, Altered expression of miR-24, miR-126 and miR-365 does not affect viability of childhood TCF3-rearranged leukemia cells, Leukemia 28 (5) (2014) 1008-1014.

[135] Y. Tan, K. Lin, Y. Zhao, Q. Wu, D. Chen, J. Wang, Y. Liang, J. Li, J. Hu, H. Wang, Y. Liu, S. Zhang, W. He, Q. Huang, X. Hu, Z. Yao, B. Liang, W. Liao, M. Shi, Adipocytes fuel gastric cancer omental metastasis via PITPNC1-mediated fatty acid metabolic reprogramming, Theranostics 8 (19) (2018) 5452-5468.

[136] Y. Tan, R. Shao, J. Li, H. Huang, Y. Wang, M. Zhang, J. Cao, J. Zhang, J. Bu, PITPNC1 fuels radioresistance of rectal cancer by inhibiting reactive oxygen species production, Ann Transl Med 8 (4) (2020) 126.

[137] D.M. Greenawalt, S.K. Sieberts, M.C. Cornelis, C.J. Girman, H. Zhong, X. Yang, J. Guinney, L. Qi, F.B. Hu, Integrating genetic association, genetics of gene expression, and single nucleotide polymorphism set analysis to identify susceptibility Loci for type 2 diabetes mellitus, Am. J. Epidemiol. 176 (5) (2012) 423-430.

[138] J.Z. Liu, Y. Erlich, J.K. Pickrell, Case-control association mapping by proxy using family history of disease, Nat. Genet. 49 (2017) 325.

[139] T.G. Ashlin, N.J. Blunsom, M. Ghosh, S. Cockcroft, J. Rihel, Pitpncla regulates zebrafish sleep and wake behavior through modulation of insulin-like growth factor signaling, Cell Rep. 24 (6) (2018) 1389-1396.

[140] B. Larijani, V. Allen-Baume, C.P. Morgan, M. Li, S. Cockcroft, EGF regulation of PITP dynamics is blocked by inhibitors of phospholipase C and of the Ras-MAP kinase pathway, Curr. Biol. 13 (1) (2003) 78-84.

[141] P.A. van Paridon, T.W.J. Gadella Jr., K.W.A. Wirtz, The effect of polyphosphoinositides and phosphatidic acid on the phosphatidylinositol transfer protein from bovine brain: a kinetic study, Biochim. Biophys. Acta 943 (1988) 76-86.

[142] H. Komatsu, B. Bouma, K.W.A. Wirtz, T.F. Taraschi, N. janes, Activity of phosphatidylinositol transfer protein is sensitive to ethanol and membrane curvature, Biochem. J. 348 (2000) 667-673.

[143] D.A. Sweeney, A. Siddhanta, D. Shields, Fragmentation and re-assembly of the Golgi apparatus in vitro. A requirement for phosphatidic acid and phosphatidylinositol 4,5-bisphosphate synthesis, J. Biol. Chem. 277 (4) (2002) 3030-3039.

[144] A. Siddhanta, J.M. Backer, D. Shields, Inhibition of phosphatidic synthesis alters the structure of the Golgi apparatus and inhibits secretion in endocrine cells, J. Biol. Chem. 275 (2000) 12023-12031.

[145] Z. Freyberg, A. Siddhanta, D. Shields, "Slip, sliding away": phospholipase D and the Golgi apparatus, Trends Cell Biol. 13 (10) (2003) 540-546.

[146] C. Riebeling, A.J. Morris, D. Shields, Phospholipase D in the Golgi apparatus, Biochim. Biophys. Acta 1791(9) (2009) 876-80.

[147] D. Cai, M. Zhong, R. Wang, W.J. Netzer, D. Shields, H. Zheng, S.S. Sisodia, D. A. Foster, F.S. Gorelick, H. Xu, P. Greengard, Phospholipase D1 corrects impaired betaAPP trafficking and neurite outgrowth in familial Alzheimer's disease-linked presenilin-1 mutant neurons, Proc. Natl. Acad. Sci. U. S. A. 103 (6) (2006) 1936-1940.

[148] E. Tanguy, P. Coste de Bagneaux, N. Kassas, M.R. Ammar, Q. Wang, A.M. Haeberle, J. Raherindratsara, L. Fouillen, P.Y. Renard, M. Montero-Hadjadje, S. Chasserot-Golaz, S. Ory, S. Gasman, M.F. Bader, N. Vitale, Mono- and polyunsaturated phosphatidic acid regulate distinct steps of regulated exocytosis in neuroendocrine cells, Cell Rep. 32(7) (2020) 108026 